\title{
ORTHOGONAL SUBSETS OF ROOT SYSTEMS AND THE ORBIT METHOD
}

\author{
M. V. IGNAT ${ }^{\prime} \mathrm{EV}$
}

\begin{abstract}
Let $k$ be the algebraic closure of a finite field, $G$ a Chevalley group over $k$, $U$ the maximal unipotent subgroup of $G$. To each orthogonal subset $D$ of the root system of $G$ and each set $\xi$ of $|D|$ nonzero scalars in $k$ one can assign the coadjoint orbit of $U$. It is proved that the dimension of such an orbit does not depend on $\xi$. An upper bound for this dimension is also given in terms of the Weyl group.
\end{abstract}

\section{$\S 0$. INTRODUCTION}

0.1. In studying irreducible complex representations of finite unipotent groups, the main tool is the orbit method. It was created by Kirillov for nilpotent Lie groups over $\mathbb{R}$, see [12] and [13, and then adapted by Kazhdan [11] to the case of finite groups (see also [14] and the paper [3], where the theory of $\ell$-adic sheaves for unipotent groups was explained). Here we consider the groups $U(q)$ and $U$, the maximal unipotent subgroups of Chevalley groups over a finite field $\mathbb{F}_{q}$ and its algebraic closure, respectively.

The orbit method establishes a bijection between the set of equivalence classes of irreducible representations of $U(q)$ and the set of orbits of the coadjoint representation of $U(q)$. Note that many questions about representations can be interpreted in terms of orbits, and that the problem of giving a complete description of orbits remains unsolved and seems to be very difficult. On the other hand, much information about some special types of orbits, representations, and characters is known.

In particular, a description is known for the regular orbits (i.e., those of maximal dimension) of the group $\mathrm{UT}_{n}$ of all unipotent triangular matrices of size $n \times n$; see [13]. The subregular orbits (i.e., orbits of second maximal dimension) and the corresponding characters 1 were described in [7] and [8]. As a generalization, Panov considered orbits of the group $\mathrm{UT}_{n}$ associated with involutions in the symmetric group. In [16, he obtained a formula for the dimension of such an orbit.

It is well known that the group $\mathrm{UT}_{n}$ corresponds to the root system of type $A_{n-1}$. In order to generalize the results of Panov, we introduced the concept of orbits associated with orthogonal subsets of root systems. In the paper [10, we studied these orbits for the case of classical root systems. (For some special orthogonal subsets of the root systems of types $B_{n}$ and $D_{n}$, we also obtained a formula involving the corresponding irreducible characters; see [9, Theorem 3.8].)

Our main goal in this paper is to extend the results of [10] to the general case of an arbitrary root system, not only the classical one. The paper is organized as follows. In the remaining part of this section, we give the necessary definitions and formulate

\footnotetext{
2010 Mathematics Subject Classification. Primary 17B22.

Key words and phrases. Orthogonal subsets of root systems, coadjoint orbits.

${ }^{1}$ The description of the irreducible character corresponding to a given orbit is itself a nontrivial problem; see, e.g., the papers of André and Neto [1] and 2] for the description of the so-called supercharacters. In this paper, we concentrate on orbits, not on characters.
} 
the main result (see Theorem 0.2). In $\S 1$, we prove some preliminary technical lemmas and consider some important examples. In $\S 2$, we prove the main theorem for simply laced root systems (see Propositions 2.4 and 2.5). In $\S 3$, we prove the main theorem for multiply laced root systems.

The author is sincerely grateful to his scientific adviser, professor A. N. Panov, for permanent attention to this work and to the referee for valuable remarks.

0.2. In this subsection, we briefly recall some basic facts concerning Chevalley groups over finite fields. We also give some definitions needed in order to formulate the main result.

Let $\Phi$ be a reduced root system, $\Delta \subset \Phi$ a subset of fundamental roots, $\Phi^{+}$and $\Phi^{-}$ the corresponding subsets of positive and negative roots, respectively (see [4]). As usual, we denote by $W=W(\Phi)$ the Weyl group of the root system $\Phi$. Let $r_{\alpha} \in W$ be the reflection on the hyperplane orthogonal to a given root $\alpha \in \Phi$.

Let $p$ be a prime, $\mathbb{F}_{q}$ the field with $q=p^{r}$ elements for some $r \geq 1, k=\overline{\mathbb{F}}_{q}$ its algebraic closure. Let $G(q)=G_{\mathrm{sc}}\left(\Phi, \mathbb{F}_{q}\right)$ (respectively, $G=G_{\mathrm{sc}}(\Phi, k)$ ) be the simply connected Chevalley group over the field $\mathbb{F}_{q}$ (respectively, over $k$ ) with the root system $\Phi$ (for the precise definitions, see the classical book [18 and also [17]). Recall that there exists a so-called Chevalley basis of the Lie algebra $\mathfrak{g}$ of the group $G$. In particular, this basis contains the root vectors $\left\{e_{\alpha}, \alpha \in \Phi^{+}\right\}$satisfying $\left[e_{\alpha}, e_{\beta}\right]=N_{\alpha \beta} e_{\alpha+\beta}$, where the $N_{\alpha \beta}$ are what is called Chevalley structure constants (here we set $N_{\alpha \beta}=0$ if $\alpha+\beta \notin \Phi$ ).

The subspace $\mathfrak{u}=\sum_{\alpha \in \Phi^{+}} k e_{\alpha}$ is a nilpotent Lie subalgebra of $\mathfrak{g}$. From now on we assume that $p$ is not less than the Coxeter number of the root system $\Phi$. This implies that $\left[x_{1},\left[x_{2},\left[\ldots,\left[x_{p-1}, x_{p}\right] \ldots\right]\right]\right]=0$ for all $x_{i} \in \mathfrak{u}$, so that the orbit method applies; see [3, Theorem 2.2 and $\S 3.3]$.

Since $p$ is sufficiently large, the exponential map exp $: \mathfrak{u} \rightarrow G$ is well defined. Its image $U$ is a maximal unipotent subgroup of $G$, and the map exp : $\mathfrak{u} \rightarrow U$ is a bijection. Next, $U$ is generated as a subgroup of $G$ by all root subgroups corresponding to the positive roots in $\Phi$, and $\mathfrak{u}$ is the Lie algebra of $U$.

Thus, the group $U$ acts on $\mathfrak{u}$ via the adjoint representation. The dual representation of $U$ in the space $\mathfrak{u}^{*}$ of all $k$-linear functions on $\mathfrak{u}$ is said to be coadjoint. It can be seen that the coadjoint action has the form

$$
\exp (y) \cdot f(x)=f\left(\exp \operatorname{ad}_{-y} x\right), \quad x, y \in \mathfrak{u}, f \in \mathfrak{u}^{*} .
$$

Here $\operatorname{ad}_{y} x=[y, x]$; since $\operatorname{ad}_{y}$ is a nilpotent linear operator on $\mathfrak{u}$, the map $\exp \operatorname{ad}_{y}=$ $\sum_{i=0}^{\infty} \operatorname{ad}_{y}^{i} / i !: \mathfrak{u} \rightarrow U$ is well defined.

In a similar way, we can define the algebra $\mathfrak{u}(q) \subset \mathfrak{g}(q)$, the group $U(q)$ and its coadjoint representation in the space $\mathfrak{u}^{*}(q)=(\mathfrak{u}(q))^{*}$. We fix an embedding $\mathbb{F}_{q} \subset k$. Then $\mathfrak{u}(q)$ can be embedded canonically in $\mathfrak{u}$. In the paper we concentrat 2 on coadjoint orbits of the group $U$, not of $U(q)$.

Now we shall give the main definition. Let $D$ be a subset of $\Phi^{+}$consisting of pairwise orthogonal roots; such a $D$ is said to be orthogonal. Let $\xi=\left(\xi_{\beta}\right)_{\beta \in D}$ be a set of nonzero scalars in $k$. Denoting by $\left\{e_{\alpha}^{*}\right\}$ the basis of $\mathfrak{u}^{*}$ dual to the basis $\left\{e_{\alpha}, \alpha \in \Phi^{+}\right\}$of the algebra $\mathfrak{u}$, we set

$$
f=f_{D, \xi}=\sum_{\beta \in D} \xi_{\beta} e_{\beta}^{*} \in \mathfrak{u}^{*}
$$

\footnotetext{
${ }^{2}$ The set of irreducible representations of the group $U(q)$ is in one-to-one correspondence with the set of coadjoint orbits of $U(q)$, but a lot of questions about representations can be interpreted in terms of orbits of the group $U$; see [1].
} 
Definition 0.1. We say that the orbit $\Omega=\Omega_{D, \xi} \subset \mathfrak{u}^{*}$ of the element $f$ under the coadjoint action of the group $U$ is associated with the subset $D$. The element $f$ is called the canonical form on the orbit $\Omega$.

Note that many important examples deal with orbits associated with orthogonal subsets; see Subsection 1.4.

0.3. To formulate the main result, we need some facts concerning involutions in the Weyl group of the root system $\Phi$. Namely, for a given orthogonal subset $D \subset \Phi^{+}$, we put

$$
\sigma=\sigma_{D}=\prod_{\beta \in D} r_{\beta} \in W
$$

(the commuting reflections $r_{\beta}$ are taken in any fixed order). Obviously, $\sigma$ is an involution, i.e., an element of order two of the group $W$.

To each element $w \in W$ we can assign two numbers $l(w)$ and $s(w)$. By definition, $l(w)$ (respectively, $s(w)$ ) is the length of a reduced (the shortest) expression of $w$ as a product of simple (respectively, arbitrary) reflections. Then $s(\sigma)=|D|$. It is well known that $l(\sigma)=\left|\Phi_{\sigma}\right|$, where $\Phi_{\sigma}=\left\{\alpha \in \Phi^{+} \mid \sigma \alpha<0\right\}$. As usual, $\alpha>0$ means that $\alpha \in \Phi^{+}$, and $\alpha<0$ means that $\alpha \in \Phi^{-}$. Furthermore, by $<$we denote the usual partial order on $\Phi$ : by definition, $\alpha>\beta$ (or $\beta<\alpha$ ) if $\alpha-\beta$ is a sum of positive roots.

Now everything is ready to formulate the main theorem. Since $\Omega$ is an irreducible affine variety (see [5. Proposition 8.2] and [19, Proposition 2.5]), one can ask how to compute $\operatorname{dim} \Omega$, the dimension of $\Omega$ over $k$. (In fact, if $f$ is an element of $\mathfrak{u}^{*}(q)$ and $\Omega(q), \Omega$ are its orbits under the action of the groups $U(q), U$, respectively, then the complex dimension of the irreducible representation of $U(q)$ corresponding to the orbit $\Omega(q)$ is equal to $q^{\operatorname{dim} \Omega / 2}$; see [11.)

Theorem 0.2. Let $D$ be an orthogonal subset of $\Phi^{+}, \xi$ a set of nonzero scalars in $k$, and $\Omega=\Omega_{D, \xi}$ the orbit associated with $D$. Then $\operatorname{dim} \Omega$ does not depend on $\xi$ and is at most $l(\sigma)-s(\sigma)$.

Remark 0.3. i) This theorem proves Conjecture 1.4 in [10. Note that in many cases (e.g., for elementary orbits) $\operatorname{dim} \Omega$ is equal to $l(\sigma)-s(\sigma)$; see Subsection 1.4

ii) On the other hand, for classical groups, the difference between $\operatorname{dim} \Omega$ and $l(\sigma)-s(\sigma)$ can be computed explicitly; furthermore, a polarization of $\mathfrak{u}$ at the canonical form on $\Omega$ can be constructed; see [10, Theorems 1.1 and 1.2] (polarizations play an important role in the explicit construction of the representation corresponding to a given orbit). We do not know how to do this for an arbitrary root system.

\section{$\S 1$. LEMmAS AND EXAMPLES}

1.1. Without loss of generality we may assume $\Phi$ to be an irreducible root system. Indeed, let $\Phi=\bigcup_{i=1}^{m} \Phi_{i}$ be the decomposition of $\Phi$ into the union of its pairwise orthogonal irreducible components. Put $D=\bigcup_{i=1}^{m} D_{i}$, where $D_{i}=D \cap \Phi_{i}$. Also, put $\mathfrak{u}_{i}=\sum_{\alpha \in \Phi_{i}^{+}} k e_{\alpha}$ for all $i$, and let $\mathfrak{u}_{i}^{*}$ be the subspace of $\mathfrak{u}^{*}$ dual to the subalgebra $\mathfrak{u}_{i}$. We denote by $f_{i} \in \mathfrak{u}_{i}^{*}$ the restriction of $f$ to $\mathfrak{u}_{i}$ and by $\Omega_{i} \subset \mathfrak{u}_{i}^{*}$ the orbit of $f_{i}$ under the coadjoint action of the group $U_{i}=\exp \left(\mathfrak{u}_{i}\right)$. Finally, let $W_{i}$ denote the Weyl group of the root system $\Phi_{i}$, and let $\sigma_{i}=\sigma_{D_{i}} \in W_{i}$.

Lemma 1.1. If the claim of Theorem 0.2 holds true for all $\Omega_{i}, i=1, \ldots, m$, then so it does for the orbit $\Omega$. 
Proof. Obviously, if $\alpha \in \Phi_{i}, \beta \in \Phi_{j}$, and $i \neq j$, then $\alpha+\beta \notin \Phi$. Hence, if $x=\sum_{i=1}^{m} x_{i}$, $y=\sum_{j=1}^{m} y_{j}, x_{i}, y_{i} \in \mathfrak{u}_{i}$, then $\operatorname{ad}_{-y_{j}}^{r} x_{i}=0$ for all $j \neq i, r>0$. Since expad $\operatorname{ad}_{-y_{i}} x_{i} \in \mathfrak{u}_{i}$ for all $i$, we obtain

$$
\begin{aligned}
\exp (y) \cdot f(x) & =\left(\sum_{i=1}^{m} f_{i}\right)\left(\sum_{j=1}^{m} \operatorname{expad}_{-y_{j}} x_{j}\right) \\
& =\sum_{i=1}^{m} f_{i}\left(\exp \operatorname{ad}_{-y_{i}} x_{i}\right)=\sum_{i=1}^{m} \exp \left(y_{i}\right) \cdot f_{i}\left(x_{i}\right) .
\end{aligned}
$$

Thus, the maps

$$
\begin{aligned}
& \Omega_{1} \times \cdots \times \Omega_{m} \rightarrow \Omega:\left(\lambda_{1}, \ldots, \lambda_{m}\right) \mapsto \lambda_{1}+\cdots+\lambda_{m} \text { and } \\
& \Omega \rightarrow \Omega_{1} \times \cdots \times \Omega_{m}: \lambda \mapsto\left(\left.\lambda\right|_{\mathfrak{u}_{1}}, \ldots,\left.\lambda\right|_{\mathfrak{u}_{m}}\right)
\end{aligned}
$$

are isomorphisms of affine varieties that are inverse to each other.

Suppose that Theorem 0.2 is valid for all $\Omega_{i}$. Let $\xi_{i}=\left(\xi_{\beta}\right)_{\beta \in D_{i}}$. Since $\Omega_{i}$ coincides with $\Omega_{D_{i}, \xi_{i}}, \operatorname{dim} \Omega$ does not depend on $\xi$. On the other hand, if $i \neq j$, then $\left.r_{\beta}\right|_{\Phi_{j}}=\operatorname{id}_{\Phi_{j}}$ for all $\beta \in D_{i}$, whence $l(\sigma)=\sum_{i=1}^{m} l\left(\sigma_{i}\right)$. Finally, $s(\sigma)=|D|=\left|\bigcup_{i=1}^{m} D_{i}\right|=\sum_{i=1}^{m}\left|D_{i}\right|=$ $\sum_{i=1}^{m} s\left(\sigma_{i}\right)$. This concludes the proof.

In what follows, we assume that $\Phi$ is irreducible.

1.2. Sometimes, two orbits associated with different orthogonal subsets coincide. To give the precise statement, we need to introduce the important concept of singular roots.

Definition 1.2. Let $\beta \in \Phi^{+}$be a positive root. Roots $\alpha, \gamma \in \Phi^{+}$are called $\beta$-singular if $\alpha+\gamma=\beta$. The set of all $\beta$-singular roots is denoted by $S(\beta)$.

Of course, it is easy to describe the set of all $\beta$-singular roots for a given root $\beta$ (see [10, formula (2)] for the case of classical groups).

Suppose that there exist $\beta_{0}, \beta_{1} \in D$ such that $\beta_{0} \in S\left(\beta_{1}\right)$. Put $D^{\prime}=D \backslash\left\{\beta_{0}\right\}$, $\xi^{\prime}=\xi \backslash\left\{\xi_{\beta_{0}}\right\}, f^{\prime}=f_{D^{\prime}, \xi^{\prime}}$. Let $\Omega^{\prime}$ be the orbit of $f^{\prime}$.

Lemma 1.3. The orbit $\Omega$ coincide $\rrbracket^{3}$ with the orbit $\Omega^{\prime}$.

Proof. Suppose $\beta_{1}=\beta_{0}+\alpha$. Then $\|\alpha\|^{2}$ is equal to $\left\|\beta_{0}\right\|^{2}+\left\|\beta_{1}\right\|^{2}$. Since $\Phi$ is irreducible, we conclude that $\Phi$ is multiply laced (the root $\alpha$ is long, the $\operatorname{roots} \beta_{0}, \beta_{1}$ are short); furthermore, the square of the length of a long root is twice that of a short one. (In other words, the root system $\Phi$ is of type $B_{n}, C_{n}$, or $F_{4}$.)

Set $\widetilde{f}=\exp \left(c e_{\alpha}\right) \cdot f^{\prime}$ for some $c \in k^{*}$. We have

$$
\widetilde{f}\left(e_{\gamma}\right)=f^{\prime}\left(e_{\gamma}\right)-c \cdot f^{\prime}\left(\operatorname{ad}_{e_{\alpha}} e_{\gamma}\right)+\frac{1}{2} c^{2} \cdot f^{\prime}\left(\operatorname{ad}_{e_{\alpha}}^{2} e_{\gamma}\right)-\cdots
$$

for a given root $\gamma \in \Phi^{+}$. Suppose $\tilde{f}\left(e_{\gamma}\right) \neq 0$. Then there exists $N \geq 0$ such that $\gamma+N \alpha \in D^{\prime}$. Of course, this is true for $\gamma=\beta_{0}$ and $N=1$, because $\beta_{0}+\alpha=\beta_{1} \in D^{\prime}$. Suppose that $N \geq 2$ and $\beta_{0}+N \alpha=\beta \in D^{\prime}$ (so that $\beta \neq \beta_{1}$ ). In this case $8\left\|\beta_{0}\right\|^{2} \leq$ $N^{2} \cdot\|\alpha\|^{2}=\left\|\beta_{0}\right\|^{2}+\|\beta\|^{2}$, a contradiction. Hence $\widetilde{f}\left(e_{\beta_{0}}\right)=-c \cdot N_{\alpha \beta_{0}} \cdot \xi_{\beta_{1}}$.

Suppose that $\gamma \neq \beta_{0}$ and $\gamma+N \alpha=\beta \in D^{\prime}$. If $N=0$, then $\gamma \in D^{\prime}$, which allows us to assume that $N \geq 1$. We see that $\gamma+(N-1) \alpha=\beta-\alpha=\beta-\beta_{1}+$ $\beta_{0}$. If $N=1$, then $\beta \neq \beta_{1}$, whence $\|\gamma\|^{2}=\left\|\beta+\beta_{0}\right\|^{2}+\left\|\beta_{1}\right\|^{2}$. But $\left\|\beta_{1}\right\|^{2}=\left\|\beta_{0}\right\|^{2}$ ( $\beta_{1}$ and $\beta_{0}$ are short), and $\left\|\beta+\beta_{0}\right\|^{2} \geq 2\left\|\beta_{0}\right\|^{2}$ (the roots $\beta, \beta_{0}$ are either equal, or orthogonal). Thus, $\|\gamma\|^{2} \geq 3\left\|\beta_{0}\right\|^{2}$, a contradiction. Hence, $N \geq 2$. On the other hand, $(\beta, \alpha)=\left(\beta, \beta_{1}\right)-\left(\beta, \beta_{0}\right)$ and $\gamma=\beta-N \alpha$. If $\beta \neq \beta_{1}$, then $(\beta, \alpha) \leq 0$, so that

${ }^{3}$ Cf. 10 Proposition 2.1]. 
$\|\gamma\|^{2}=\|\beta\|^{2}+N^{2} \cdot\|\alpha\|^{2}-2 N \cdot(\beta, \alpha) \geq 4\left\|\alpha^{2}\right\|$, a contradiction. But if $\beta=\beta_{1}$, then $\|\gamma\|^{2}=\left\|N \beta_{0}+(1-N) \beta_{1}\right\|^{2}=\left(N^{2}+(1-N)^{2}\right)\left\|\beta_{0}\right\|^{2} \geq 5\left\|\beta_{0}\right\|^{2}$, a contradiction.

We conclude that $\widetilde{f}\left(e_{\gamma}\right)=f^{\prime}\left(e_{\gamma}\right)=f\left(e_{\gamma}\right)$ for all $\gamma \neq \beta_{0}$. Hence, if $c=-\xi_{\beta_{0}} /\left(N_{\alpha \beta_{0}} \cdot \xi_{\beta_{1}}\right)$, then $\widetilde{f}$ coincides with $f$. By definition, $\widetilde{f} \in \Omega^{\prime}$, whence $\Omega^{\prime}=\Omega$, as required.

From now on and till the end of the paper, we assume that $S(\beta) \cap D=\varnothing$ for all $\beta \in D$.

1.3. To prove the main theorem for simply laced root systems, we need some more preparation. Let $\eta, \eta^{\prime}, \eta_{i}, \theta, \theta^{\prime}, \theta_{j}, \psi, \psi^{\prime}, \psi_{l}, \psi_{l}^{\prime}$ be distinct positive roots; assume the roots $\eta, \eta^{\prime}, \eta_{i}$ to be pairwise orthogonal and assume the root $\eta$ to be maximal among all $\eta$ 's with respect to the usual order on $\Phi$. Consider the following cases:

$$
\begin{aligned}
& \text { 1. } \eta=\theta+\psi=\theta^{\prime}+\psi^{\prime}, \quad \text { 2. } \quad \eta=\theta+\psi, \quad \text { 3. } \eta=\theta+\psi \text {, } \\
& \eta_{1}=\theta_{1}+\psi, \quad \eta^{\prime}=\theta^{\prime}+\psi, \quad \eta_{1}=\theta_{1}+\psi, \\
& \eta_{2}=\theta_{2}+\psi, \quad \eta_{1}=\theta+\psi_{1}, \quad \eta_{2}=\theta+\psi_{2}, \\
& \eta^{\prime}=\theta_{1}+\psi^{\prime}, \quad \eta_{2}=\theta+\psi_{2}, \quad \eta_{3}=\theta_{1}+\psi_{3}, \\
& \text { 4. } \eta=\theta+\psi=\theta_{1}^{\prime}+\psi_{1}^{\prime} \text {, } \\
& \eta_{1}=\theta_{1}+\psi=\theta_{1}^{\prime}+\psi^{\prime}, \\
& \eta^{\prime}=\theta+\psi^{\prime}=\theta_{1}+\psi_{1}^{\prime} \text {, } \\
& \left\{\theta, \theta_{1}, \theta_{1}^{\prime}, \psi, \psi^{\prime}, \psi_{1}^{\prime}\right\} \cap S\left(\eta_{2}\right) \neq \varnothing .
\end{aligned}
$$

Definition 1.4. A set of positive roots satisfying the conditions of type $1,2,3$, or 4 is called nonadmissible.

Lemma 1.5. There are no nonadmissible subsets in $D_{5}^{+}$.

Proof. Straightforward.

1.4. Before the proof of the main theorem, we consider some examples of orbits associated with orthogonal subsets. First, we consider the case where $\Phi=A_{n-1}$ (i.e., $U=\mathrm{UT}_{n}$, the unitriangular group). It is convenient to identify $A_{n-1}^{+}$with the subset of $\mathbb{R}^{n}$ of the form $\left\{\varepsilon_{i}-\varepsilon_{j}, 1 \leq i<j \leq n\right\}$ (by $\left\{\varepsilon_{i}\right\}_{i=1}^{n}$ we denote the standard basis of $\mathbb{R}^{n}$ ). The Weyl group of $A_{n-1}$ is isomorphic to the symmetric group $S_{n}$ on $n$ letters.

Example 1.6. i) Let $D=D_{\text {reg }}=\left\{\varepsilon_{1}-\varepsilon_{n}, \varepsilon_{2}-\varepsilon_{n-1}, \ldots, \varepsilon_{n_{1}}-\varepsilon_{n_{2}}\right\}$, where $n_{1}=[n / 2]$, $n_{2}=n-n_{1}+1$. Then $\sigma$ is the longest element of the Weyl group $W$ and $\Phi_{\sigma}=\Phi^{+}$(i.e., $\sigma(\alpha)<0$ for all positive roots $\alpha$ ). Then the orbit $\Omega$ is regular; i.e., it has the maximal dimension among all coadjoint orbits. The dimension of $\Omega$ equals

$$
\operatorname{dim} \Omega=l(\sigma)-s(\sigma)=2 \mu(n), \mu(n)=(n-2)+(n-4)+\cdots .
$$

ii) Now let

$$
D=D_{\text {sreg }}=\left(D_{\text {reg }} \backslash\left\{\varepsilon_{i}-\varepsilon_{n-i+1}, \varepsilon_{i+1}-\varepsilon_{n-i}\right\}\right) \cup\left\{\varepsilon_{i}-\varepsilon_{n-i}, \varepsilon_{i+1}-\varepsilon_{n-i+1}\right\} .
$$

In this case, the orbit $\Omega$ is subregular, i.e., has the second maximal $\operatorname{dimension} \operatorname{dim} \Omega=$ $l(\sigma)-s(\sigma)=2 \mu(n)-2 ;$ see [7, $\S 3]$.

Example 1.7. Let $\Phi$ be an arbitrary root system. Suppose that $|D|=1$. Then the orbit $\Omega$ is said to be elementary. It is easily seen that $\operatorname{dim} \Omega=|S(\beta)|$; see [15, $\S 4$ ]. It is straightforward to check that $l(\sigma)-s(\sigma)$ coincides with $|S(\beta)|$ (see [10, §4] for the case of classical groups). 
Example 1.8. On the other hand, if $\Phi=B_{3}$ (recall that $B_{3}^{+}=\left\{\varepsilon_{i}, \varepsilon_{i} \pm \varepsilon_{j}, 1 \leq i<j\right.$ $\leq 3\})$ and $D=\left\{\varepsilon_{1}, \varepsilon_{2}+\varepsilon_{3}\right\}$, then the dimension of $\Omega$ is smaller than $l(\sigma)-s(\sigma)$, because $\operatorname{dim} \Omega=4$ and $l(\sigma)-s(\sigma)=6$ (see [9] or [10]).

\section{§2. Simply LaCED RoOT SYSTEMS}

2.1. Throughout this section, $\Phi$ is a simply laced root system, i.e., all roots in $\Phi$ have the same length. (In other words, $\Phi$ is of type $A_{n}, D_{n}, E_{6}, E_{7}$, or $E_{8}$.) Without loss of generality, suppose that the length of a root in $\Phi$ equals 1 . Then the inner product of two nonorthogonal roots in $\Phi$ equals either \pm 1 or $\pm 1 / 2$. Moreover, suppose $\alpha, \beta \in \Phi^{+}$. Then $(\beta, \alpha)=1 / 2$ if and only if either $\alpha \in S(\beta)$ or $\beta \in S(\alpha)$; in this case, $r_{\beta} \alpha=\alpha-\beta$. On the other hand, $(\alpha, \beta)=-1 / 2$ if and only if $\alpha+\beta \in \Phi^{+}$; in this case, $r_{\beta} \alpha=\alpha+\beta$.

As above, let $D$ be an orthogonal subset of $\Phi^{+}, \xi$ a set of nonzero scalars in $k, \Omega=\Omega_{D, \xi}$ the associated coadjoint orbit, and $f$ the canonical form on $\Omega$. First, we prove that the dimension of the orbit $\Omega$ does not exceed $l(\sigma)-s(\sigma)$. The proof is by induction on the rank of $\Phi$. The base ( $\operatorname{rk} \Phi=1$, i.e., $\Phi=A_{1}$ ) is straightforward. To perform the inductive step, it suffices to prove the claim only for irreducible root systems of a given rank, as Lemma 1.1 shows.

For the case where $|D|=1$ (i.e., the case of elementary orbits), there is nothing to prove; see Example 1.7. Suppose $|D|>1$. We pick a root $\beta$ maximal among all roots in $D$ and put $\widetilde{D}=D \backslash\{\beta\}$. In order to use the inductive hypothesis, we shall define the root system of rank less than the rank of $\Phi$. Precisely, put $\mathcal{A}=\left\{\alpha \in \Phi^{+} \mid(\alpha, \beta) \neq 0\right\}$ and $\widetilde{\Phi}= \pm \widetilde{\Phi}^{+}$, where

$$
\widetilde{\Phi}^{+}=\Phi^{+} \backslash \mathcal{A}=\left\{\alpha \in \Phi^{+} \mid(\alpha, \beta)=0\right\} .
$$

The following lemma is obvious.

Lemma 2.1. The set $\widetilde{\Phi}$ is a root system.

By construction, $\operatorname{rk} \widetilde{\Phi}<\operatorname{rk} \Phi$. Obviously, $\widetilde{D}=D \cap \widetilde{\Phi}^{+}$. Denote by $\widetilde{\mathfrak{u}}$ the subalgebra of $\mathfrak{u}$ spanned by all vectors $e_{\alpha}, \alpha \in \widetilde{\Phi}^{+}$. Put $\widetilde{f}=\left.f\right|_{\widetilde{\mathfrak{u}}} \in \widetilde{\mathfrak{u}}^{*} \subset \mathfrak{u}^{*}$, so that $f=\xi_{\beta} e_{\beta}^{*}+\widetilde{f}$. Putting also $\widetilde{U}=\exp (\widetilde{\mathfrak{u}}), \widetilde{\xi}=\xi \backslash\left\{\xi_{\beta}\right\}$, we let $\widetilde{\Omega}=\Omega_{\widetilde{D}, \widetilde{\xi}} \subset \widetilde{\mathfrak{u}}^{*}$ be the coadjoint orbit of the group $\widetilde{U}$ associated with $\widetilde{D}$. Then $\widetilde{f}$ is the canonical form on the orbit $\widetilde{\Omega}$.

Finally, let $\widetilde{\sigma}$ be the involution in the Weyl group $\widetilde{W}$ of the root system $\widetilde{\Phi}$ corresponding to the subset $\widetilde{D}$. By the inductive assumption, $\operatorname{dim} \widetilde{\Omega}$ does not exceed $l(\widetilde{\sigma})-s(\widetilde{\sigma})$. Obviously, $s(\sigma)=s(\widetilde{\sigma})+1$; thus, it remains to compare $l(\sigma)$ with $l(\widetilde{\sigma})$.

2.2. Let $\mathfrak{a}$ be the radical of the bilinear form $x, y \mapsto f([x, y]), x, y \in \mathfrak{u}$, i.e.,

$$
\mathfrak{a}=\mathfrak{r a d}_{\mathfrak{u}} f=\{x \in \mathfrak{u} \mid f([x, y])=0 \text { for all } y \in \mathfrak{u}\} .
$$

It is well known that $\operatorname{dim} \Omega=\operatorname{codim}_{\mathfrak{u}} \mathfrak{a}=\left|\Phi^{+}\right|-\operatorname{dim} \mathfrak{a} ;$ see [2, §3]. Similarly, let $\widetilde{\mathfrak{a}}=\mathfrak{r a d}_{\mathfrak{u}} \tilde{f}$ be the radical of the bilinear form $x, y \mapsto \widetilde{f}([x, y]), x, y \in \widetilde{\mathfrak{u}}$. Then $\operatorname{dim} \widetilde{\Omega}=\operatorname{codim}_{\mathfrak{u}} \widetilde{\mathfrak{a}}=$ $\left|\widetilde{\Phi}^{+}\right|-\operatorname{dim} \tilde{\mathfrak{a}}$. Put $\mathfrak{u}_{\mathcal{A}}=\sum_{\alpha \in \mathcal{A}} k e_{\alpha}$ (so that $\mathfrak{u}=\mathfrak{u}_{\mathcal{A}} \oplus \widetilde{\mathfrak{u}}$ as vector spaces) and $\mathfrak{b}=\mathfrak{a} \cap \mathfrak{u}_{\mathcal{A}}$.

Lemma 2.2. The subalgebra $\mathfrak{a}$ coincides with the direct sum of its subspaces $\mathfrak{b}$ and $\widetilde{\mathfrak{a}}$, i.e., $\mathfrak{a}=\mathfrak{b} \oplus \tilde{\mathfrak{a}}$.

Proof. i) For any $x=\sum_{\alpha \in \Phi^{+}} b_{\alpha} e_{\alpha} \in \mathfrak{u}$, let $\operatorname{Supp}(x)=\left\{\alpha \in \Phi^{+} \mid b_{\alpha} \neq 0\right\}$. Let $\mathfrak{r}=\mathfrak{r a d}_{\mathfrak{u}} \xi_{\beta} e_{\beta}^{*}=\mathfrak{r a d} \mathfrak{d}_{\mathfrak{u}} e_{\beta}^{*}=\left\langle e_{\alpha} \mid \alpha \in \Phi^{+} \backslash S(\beta)\right\rangle_{k} \supset \widetilde{\mathfrak{u}}$. Suppose that there exists $x \in \tilde{\mathfrak{a}}$ such that $x \notin \mathfrak{a} \cap \widetilde{\mathfrak{u}}$. Then there exists $y \in \mathfrak{u}$ such that $f([x, y]) \neq 0$. Since $x \in \tilde{\mathfrak{u}} \subset \mathfrak{r}$, we see that $\xi_{\beta} e_{\beta}^{*}([x, y])=0$, whence $\widetilde{f}([x, y]) \neq 0$. Hence, there exists $\alpha \in \operatorname{Supp}(x)$ and $\gamma \in \mathcal{A} \cap \operatorname{Supp}(y)$ such that $\alpha+\gamma=\widetilde{\beta} \in \widetilde{D}$. Using the orthogonality of the subset $D$ and 
the fact that $\gamma \in \mathcal{A}$, we get $(\alpha, \beta)=(\widetilde{\beta}-\gamma, \beta)=(\widetilde{\beta}, \beta)-(\gamma, \beta)=-(\gamma, \beta) \neq 0$. This contradicts the choice of $\alpha \in \widetilde{\Phi}^{+}$. Thus, $x \in \mathfrak{a} \cap \widetilde{\mathfrak{u}}$, so $\widetilde{\mathfrak{a}} \subset \mathfrak{a} \cap \widetilde{\mathfrak{u}} \subset \mathfrak{a}$.

ii) On the other hand, let $x=y+z \in \mathfrak{a}$, where $y \in \mathfrak{u}_{\mathcal{A}}, z \in \widetilde{\mathfrak{u}}$. If $\gamma \in \widetilde{\Phi}^{+}$, then $\gamma \notin S(\beta)$ and $\alpha+\gamma \in \mathcal{A}$ for all $\alpha \in \mathcal{A}$. Therefore, $f\left(\left[x, e_{\gamma}\right]\right)=\xi_{\beta} e_{\beta}^{*}\left(\left[x, e_{\gamma}\right]\right)+\widetilde{f}\left(\left[y, e_{\gamma}\right]\right)+\widetilde{f}\left(\left[z, e_{\gamma}\right]\right)=$ $\widetilde{f}\left(\left[z, e_{\gamma}\right]\right)=0$, i.e., $z \in \widetilde{\mathfrak{a}}$. In accordance with step i), we have $z \in \mathfrak{a} \cap \tilde{\mathfrak{u}} \subset \mathfrak{a}$. Consequently, $y=x-z \in \mathfrak{a}$, whence $y \in \mathfrak{a} \cap \mathfrak{u}_{\mathcal{A}}=\mathfrak{b}$ and $\mathfrak{a}=\mathfrak{b}+\widetilde{\mathfrak{a}}$. But $\mathfrak{b} \cap \tilde{\mathfrak{a}}=0$, so that the sum is direct. This concludes the proof.

2.3. To prove the inequality $\operatorname{dim} \Omega \leq l(\sigma)-s(\sigma)$, we need the following key observation.

Lemma 2.3. We have the inequality $\#\{\alpha \in \mathcal{A} \mid \sigma \alpha>0\}+1 \leq \operatorname{dim} \mathfrak{b}$.

Proof. Let $\widetilde{\mathcal{A}}=\{\alpha \in \mathcal{A} \mid \sigma \alpha>0\} \cup\{\beta\}$ (clearly, $\sigma \beta=-\beta<0$ ). It suffices to construct a linearly independent set $\left\{x_{\alpha}\right\}_{\alpha \in \widetilde{\mathcal{A}}} \subset \mathfrak{b}$. Since $(\beta, \beta)=1$ and $\beta$ is not singular to any root in $D$, it follows that $\beta \in \mathcal{A}$ and $x_{\beta}=e_{\beta} \in \mathfrak{b}$.

It is convenient to split the set $\widetilde{\mathcal{A}}$ into the union $\widetilde{\mathcal{A}}=\mathcal{A}^{+} \cup \mathcal{A}^{-} \cup\{\beta\}$, where

$$
\begin{aligned}
& \mathcal{A}^{+}=\{\alpha \in \mathcal{A} \mid \sigma \alpha>0 \text { and }(\alpha, \beta)>0\}, \\
& \mathcal{A}^{-}=\{\alpha \in \mathcal{A} \mid \sigma \alpha>0 \text { and }(\alpha, \beta)<0\} .
\end{aligned}
$$

We consider two different cases, $\alpha \in \mathcal{A}^{-}$and $\alpha \in \mathcal{A}^{+}$.

i) First, let $\alpha \in \mathcal{A}^{-}$, i.e., $(\alpha, \beta)=-1 / 2<0$ and $\sigma \alpha>0$. Suppose $\alpha$ is singular to the roots $\beta_{1}, \ldots, \beta_{l} \in D$ and is not singular to any other root in $D$. Put $\gamma_{i}=\beta_{i}-\alpha \in \Phi^{+}$ for all $i=1, \ldots, l$. Then

$$
\left(\beta, \gamma_{i}\right)=\left(\beta, \beta_{i}-\alpha\right)=-(\beta, \alpha)=1 / 2 .
$$

Thus, either $\gamma_{i} \in S(\beta)$ or $\beta \in S\left(\gamma_{i}\right)$. But if the second case occurs, then $\beta<\beta_{i}$, which contradicts the choice of the $\operatorname{root} \beta$. So, $\gamma_{i} \in S(\beta)$ for all $i$. Put $\alpha_{i}=\beta-\gamma_{i} \in \Phi^{+}$, $i=1, \ldots, l$.

Now we set $b_{i}=-\left(\xi_{\beta_{i}} \cdot N_{\alpha \gamma_{i}}\right) /\left(\xi_{\beta} \cdot N_{\alpha_{i} \gamma_{i}}\right), i=1, \ldots, l$, and $x_{\alpha}=e_{\alpha}+\sum_{i=1}^{l} b_{i} e_{\alpha_{i}}$. Clearly, $x_{\alpha} \in \mathfrak{u}_{\mathcal{A}}$. We claim that $x_{\alpha} \in \mathfrak{a}=\mathfrak{r a d}_{\mathfrak{u}} f$. Indeed, let $\delta$ be a positive root. By definition,

$$
f\left(\left[x_{\alpha}, e_{\delta}\right]\right)=N_{\alpha \delta} \cdot f\left(e_{\alpha+\delta}\right)+\sum_{i=1}^{l} N_{\alpha_{i} \delta} \cdot f\left(e_{\alpha_{i}+\delta}\right) \cdot b_{i} .
$$

We pick a number $i$ and observe that $\alpha_{i}+\delta \notin D$ if $\delta \neq \gamma_{i}$. Indeed, suppose the contrary. Then there exists $\widetilde{\beta} \in D$ such that $\widetilde{\beta} \neq \beta$ and $\alpha_{i}+\delta=\widetilde{\beta}$. (Obviously, $\delta \neq \gamma_{i}$ is equivalent to $\widetilde{\beta} \neq \beta$.) Since $\left(\widetilde{\beta}, \alpha_{i}\right)=1 / 2$, we have $\left(\widetilde{\beta}, \gamma_{i}\right)=\left(\widetilde{\beta}, \beta-\alpha_{i}\right)=-1 / 2$. Hence, $(\widetilde{\beta}, \alpha)=\left(\widetilde{\beta}, \beta_{i}-\gamma_{i}\right)=1 / 2$. Let $\beta_{1}^{\prime}, \ldots, \beta_{s}^{\prime}$ be all the roots in $D$ that are not orthogonal to $\alpha$ except the roots $\beta, \beta_{i}, \widetilde{\beta}$. Then

$$
\sigma \alpha=\alpha+\beta-\beta_{i}+\widetilde{\beta}-2\left(\alpha, \beta_{1}^{\prime}\right) \cdot \beta_{1}^{\prime}-\cdots-2\left(\alpha, \beta_{s}^{\prime}\right) \cdot \beta_{s}^{\prime} .
$$

Since $\left(\alpha, \beta_{r}^{\prime}\right)= \pm 1 / 2$ for all $1 \leq r \leq s$, we obtain

$$
\|\sigma \alpha-\alpha\|^{2}=2-2(\sigma \alpha, \alpha)=\|\beta\|^{2}+\left\|\beta_{i}\right\|^{2}+\|\widetilde{\beta}\|^{2}+\sum_{r=1}^{s}\left\|\beta_{r}^{\prime}\right\|^{2}=3+s .
$$

We see that either $s=0$ or $s=1$, because $(\sigma \alpha, \alpha) \geq-1$. If $s=0$, then

$$
\sigma \alpha=\alpha+\beta-\beta_{i}-\widetilde{\beta}=\alpha+\left(\alpha_{i}+\gamma_{i}\right)-\left(\alpha+\gamma_{i}\right)-\left(\alpha_{i}+\delta\right)=-\delta<0 .
$$

On the other hand, $\left(\alpha, \beta_{i}\right)=1 / 2$ because $\alpha \in S\left(\beta_{i}\right)$; therefore, if $s=1$, then

$$
\begin{aligned}
(\sigma \alpha, \alpha) & =\|\alpha\|^{2}+(\beta, \alpha)-\left(\beta_{i}, \alpha\right)-(\widetilde{\beta}, \alpha)-2\left(\beta_{1}^{\prime}, \alpha\right)^{2} \\
& =1-1 / 2-1 / 2-1 / 2-1 / 2=-1,
\end{aligned}
$$


i.e., $\sigma \alpha=-\alpha<0$. By the way, $\sigma \alpha<0$. This contradicts the choice of $\alpha$. We conclude that $\alpha_{i}+\delta \notin D$ if $\delta \neq \gamma_{i}$.

Hence, if $\delta=\gamma_{i}$ for some $i$, then

$$
\begin{aligned}
f\left(\left[x_{\alpha}, e_{\delta}\right]\right) & =f\left(\left[x_{\alpha}, e_{\gamma_{i}}\right]\right)=N_{\alpha \gamma_{i}} \cdot \xi_{\beta_{i}}+N_{\alpha_{i} \gamma_{i}} \cdot \xi_{\beta} \cdot b_{i} \\
& =N_{\alpha \gamma_{i}} \cdot \xi_{\beta_{i}}-N_{\alpha_{i} \gamma_{i}} \cdot \xi_{\beta} \cdot\left(\xi_{\beta_{i}} \cdot N_{\alpha \gamma_{i}}\right) /\left(\xi_{\beta} \cdot N_{\alpha_{i} \gamma_{i}}\right) \\
& =N_{\alpha \gamma_{i}} \cdot \xi_{\beta_{i}}-N_{\alpha \gamma_{i}} \cdot \xi_{\beta_{i}}=0 .
\end{aligned}
$$

On the other hand, if $\delta \neq \gamma_{i}$ for all $i$, then $\alpha_{i}+\delta \notin D, 1 \leq i \leq l$, as above. But $\alpha+\delta \notin D$, whence $f\left(\left[x_{\alpha}, e_{\delta}\right]\right)=0$ in the case. We conclude that, for a given $\alpha \in \mathcal{A}^{-}$, the vector $x_{\alpha}$ belongs to $\mathfrak{b}=\mathfrak{a} \cap \mathfrak{u}_{\mathcal{A}}$, as required.

ii) We pass to the case where $\alpha \in \mathcal{A}^{+}$, i.e., $(\alpha, \beta)=1 / 2>0$ and $\sigma \alpha>0$. Since the Weyl group acts by orthogonal transformations, $(\beta, \sigma \alpha)=(\sigma \beta, \alpha)=(-\beta, \alpha)=-1 / 2$. This shows that $\beta+\sigma \alpha \in \Phi^{+}$. If $\beta+\sigma \alpha \in S(\widetilde{\beta})$ for some $\operatorname{root} \widetilde{\beta} \in D$, then $\beta<\widetilde{\beta}$. This contradicts the choice of $\beta$. Thus, given a root $\alpha \in \mathcal{A}^{+}$, we see that the vector $x_{\alpha}=e_{\beta+\sigma \alpha}$ belongs to $\mathfrak{b}$. Note also that $(\beta, \beta+\sigma \alpha)=1-1 / 2=1 / 2 \neq 0$, whence $\beta+\sigma \alpha \in \mathcal{A}$.

Given a root $\alpha \in \widetilde{\mathcal{A}}$, we have constructed the vector $x_{\alpha} \in \mathfrak{b}$. It remains to check that the vectors $x_{\alpha}, \alpha \in \mathcal{A}$, are linearly independent. Since $\beta+\sigma \alpha, \alpha \in \mathcal{A}^{+}$, are distinct, the corresponding vectors $x_{\alpha}=e_{\beta+\sigma \alpha}$ are linearly independent. If $\alpha \in \mathcal{A}^{-}$, then $e_{\alpha} \in \operatorname{Supp}\left(x_{\alpha}\right)$ and $\operatorname{Supp}\left(x_{\alpha}\right) \backslash\{\alpha\} \subset S(\beta)$. Consequently, $x_{\alpha}, \alpha \in \mathcal{A}^{-}$, are also linearly independent. Their union with $x_{\alpha}, \alpha \in \mathcal{A}^{+}$, are also linearly independent, because $\left(\mathcal{A}^{-} \cup S(\beta)\right) \cap\left(\beta+\sigma \mathcal{A}^{+}\right)=\varnothing$. Indeed, the inner products of $\beta$ with the roots in $\mathcal{A}^{-}$(respectively, in $\beta+\sigma \mathcal{A}^{+}$) are negative (respectively, positive), so that these subsets are disjoint. Finally, for a given $\alpha \in \mathcal{A}^{+}$, the root $\beta+\sigma \alpha \in \Phi^{+}$is not $\beta$-singular because $\beta+\sigma \alpha>\beta$. Thus, the vectors in the set $\left\{x_{\alpha}\right\}_{\alpha \in \widetilde{\mathcal{A}}}$ are linearly independent. This completes the proof.

2.4. Now we conclude the proof of the inequality $\operatorname{dim} \Omega \leq l(\sigma)-s(\sigma)$.

Proposition 2.4. Let $\Phi$ be a reduced irreducible simply laced root system, $D \subset \Phi^{+}$ an orthogonal subset, $\Omega$ an associated orbit of the group $U$, and $\sigma \in W$ the involution corresponding to $D$. Then $\operatorname{dim} \Omega \leq l(\sigma)-s(\sigma)$.

Proof. By the above (see Subsection 2.2) and the inductive hypothesis,

$$
\begin{aligned}
\operatorname{dim} \Omega & =\left|\Phi^{+}\right|-\operatorname{dim} \mathfrak{a}=\left|\widetilde{\Phi}^{+}\right|+|\mathcal{A}|-\operatorname{dim} \mathfrak{a}-\operatorname{dim} \widetilde{\mathfrak{a}}+\operatorname{dim} \widetilde{\mathfrak{a}} \\
& =\left(\left|\widetilde{\Phi}^{+}\right|-\operatorname{dim} \widetilde{\mathfrak{a}}\right)+|\mathcal{A}|-(\operatorname{dim} \mathfrak{a}-\operatorname{dim} \widetilde{\mathfrak{a}}) \\
& =\operatorname{dim} \widetilde{\Omega}+|\mathcal{A}|-(\operatorname{dim} \mathfrak{a}-\operatorname{dim} \widetilde{\mathfrak{a}}) \\
& \leq l(\widetilde{\sigma})-s(\widetilde{\sigma})+|\mathcal{A}|-(\operatorname{dim} \mathfrak{a}-\operatorname{dim} \widetilde{\mathfrak{a}})=l(\widetilde{\sigma})-s(\widetilde{\sigma})+|\mathcal{A}|-\operatorname{dim} \mathfrak{b} .
\end{aligned}
$$

It remains to check that $l(\sigma)-s(\sigma) \geq l(\widetilde{\sigma})-s(\widetilde{\sigma})+|\mathcal{A}|-\operatorname{dim} \mathfrak{b}$. But $s(\sigma)=s(\widetilde{\sigma})+1$. We also note that, since the reflection $r_{\beta}$ acts on $\widetilde{\Phi}^{+}$trivially, we have $\left|\Phi_{\sigma} \cap \widetilde{\Phi}^{+}\right|=\left|\widetilde{\Phi}_{\widetilde{\sigma}}\right|=l(\widetilde{\sigma})$ and

$$
l(\sigma)=\left|\Phi_{\sigma}\right|=\left|\Phi_{\sigma} \cap \widetilde{\Phi}^{+}\right|+\left|\Phi_{\sigma} \cap \mathcal{A}\right|=l(\widetilde{\sigma})+\#\{\alpha \in \mathcal{A} \mid \sigma \alpha<0\} .
$$

Hence, it suffices to prove that

$$
|\mathcal{A}|-\#\{\alpha \in \mathcal{A} \mid \sigma \alpha<0\}+1=\#\{\alpha \in \mathcal{A} \mid \sigma \alpha>0\}+1 \leq \operatorname{dim} \mathfrak{b},
$$

but this follows immediately from Lemma 2.3 . 
2.5. In the remainder of this section, we prove that $\operatorname{dim} \Omega$ does not depend on $\xi$. Let $\xi^{\prime}=\left(\xi_{\beta}^{\prime}\right)_{\beta \in D}$ be a set of nonzero scalars and $f^{\prime}$ the canonical form on the orbit $\Omega^{\prime}=\Omega_{D, \xi^{\prime}}$. Put $\mathfrak{a}^{\prime}=\mathfrak{r a d} \mathfrak{a}_{\mathfrak{u}} f^{\prime}$. Arguing as in the proof of Proposition 2.2, we conclude that $\mathfrak{a}^{\prime}=\mathfrak{b}^{\prime} \oplus \tilde{\mathfrak{a}}^{\prime}$ as vector spaces, where $\mathfrak{b}^{\prime}=\mathfrak{a}^{\prime} \cap \mathfrak{u}_{\mathcal{A}}, \widetilde{f}^{\prime}=\left.f^{\prime}\right|_{\mathfrak{u}}$ and $\widetilde{\mathfrak{a}}^{\prime}=\mathfrak{r a d} \mathfrak{d}_{\mathfrak{u}} \widetilde{f}^{\prime}$.

Proposition 2.5. Let $\Phi$ be a reduced irreducible simply laced root system, $D \subset \Phi^{+}$an orthogonal subset, and $\xi, \xi^{\prime}$ sets of nonzero scalars. Put $\Omega=\Omega_{D, \xi}, \Omega^{\prime}=\Omega_{D, \xi^{\prime}}$. Then $\operatorname{dim} \Omega=\operatorname{dim} \Omega^{\prime}$.

Proof. As above, $\operatorname{dim} \Omega=\operatorname{codim}_{\mathfrak{u}} \mathfrak{a}$ and $\operatorname{dim} \Omega^{\prime}=\operatorname{codim}_{\mathfrak{u}} \mathfrak{a}^{\prime}$; therefore, it remains to check that $\operatorname{dim} \mathfrak{a}=\operatorname{dim} \mathfrak{a}^{\prime}$. We proceed by induction on the rank of $\Phi$. The base $\left(\operatorname{rk} \Phi=1\right.$, i.e., $\left.\Phi=A_{1}\right)$ is evident. But $\mathfrak{a}=\mathfrak{b} \oplus \widetilde{\mathfrak{a}}, \mathfrak{a}^{\prime}=\mathfrak{b}^{\prime} \oplus \widetilde{\mathfrak{a}}^{\prime}$, and $\operatorname{dim} \widetilde{\mathfrak{a}}=\operatorname{dim} \widetilde{\mathfrak{a}}^{\prime}$ by the inductive assumption, because $\operatorname{rk} \widetilde{\Phi}<\operatorname{rk} \Phi$. Thus, it suffices to show that $\operatorname{dim} \mathfrak{b}=\operatorname{dim} \mathfrak{b}^{\prime}$.

Obviously, it suffices to prove that $\operatorname{dim} \mathfrak{b} \leq \operatorname{dim} \mathfrak{b}^{\prime}$. Set $x=\sum_{\alpha \in \mathcal{A}} x_{\alpha} e_{\alpha} \in \mathfrak{b}$. Put $y=\varphi(x)=\sum_{\alpha \in \operatorname{Supp}(x)} y_{\alpha} e_{\alpha}$. In the next subsection we prove that there exist $y_{\alpha}$ such that $y \in \mathfrak{b}^{\prime}$, and if the vectors $x_{1}, \ldots, x_{m}$ are linearly independent, then the vectors $\varphi\left(x_{1}\right), \ldots, \varphi\left(x_{m}\right)$ are also linearly independent. Applying this to an arbitrary basis $x_{i}$ of the space $\mathfrak{b}$, we obtain the result.

2.6. In this subsection, we conclude the proof of Proposition 2.5. Our first goal is to determine the coefficients $y_{\alpha}$. We set $y_{\alpha}=x_{\alpha}$ for all $\alpha \in \mathcal{A}$ except for the following four cases.

i) There exists $\alpha_{0}$ and $\gamma \in \Phi^{+}, \beta_{0} \in D$ such that

$$
\beta=\alpha+\gamma, \quad \beta_{0}=\alpha_{0}+\gamma,
$$

and $\alpha, \alpha_{0}$ are not singular to any other root in $D$. Then we put $y_{\alpha}=x_{\alpha} \cdot \xi_{\beta} / \xi_{\beta}^{\prime}$.

ii) There exist $\widetilde{\alpha}, \alpha_{0}, \gamma, \widetilde{\gamma} \in \Phi^{+}$and $\beta_{0}, \widetilde{\beta}_{0} \in D$ such that

$$
\beta=\alpha+\gamma=\widetilde{\alpha}+\widetilde{\gamma}, \quad \beta_{0}=\alpha_{0}+\gamma, \quad \widetilde{\beta}_{0}=\alpha_{0}+\widetilde{\gamma},
$$

and $\alpha, \widetilde{\alpha}, \gamma, \widetilde{\gamma}$ are not singular to any other root in $D$. We let $y_{\alpha}=x_{\alpha} \cdot\left(\xi_{\beta} \cdot \xi_{\beta_{0}}^{\prime}\right) /\left(\xi_{\beta}^{\prime} \cdot \xi_{\beta_{0}}\right)$ here. Since the above conditions are invariant under the interchanging of $\alpha$ and $\widetilde{\alpha}$, we also put $y_{\widetilde{\alpha}}=x_{\widetilde{\alpha}} \cdot\left(\xi_{\beta} \cdot \xi_{\widetilde{\beta}_{0}}^{\prime}\right) /\left(\xi_{\beta}^{\prime} \cdot \xi_{\widetilde{\beta}_{0}}\right)$.

iii) There exist $\widetilde{\alpha}, \alpha_{0}, \gamma, \widetilde{\gamma}, \gamma_{0} \in \Phi^{+}$and $\beta_{0}, \widetilde{\beta}_{0} \in D$ such that

$$
\begin{gathered}
\beta=\alpha+\gamma=\widetilde{\alpha}+\widetilde{\gamma}, \\
\beta_{0}=\alpha_{0}+\gamma=\widetilde{\alpha}+\gamma_{0}, \\
\widetilde{\beta}_{0}=\alpha+\gamma_{0}=\alpha_{0}+\widetilde{\gamma},
\end{gathered}
$$

and $\alpha, \widetilde{\alpha}, \alpha_{0}, \gamma, \widetilde{\gamma}, \gamma_{0}$ are not orthogonal to any other root in $D$. As above, we set $y_{\alpha}=x_{\alpha} \cdot\left(\xi_{\beta} \cdot \xi_{\beta_{0}}^{\prime}\right) /\left(\xi_{\beta}^{\prime} \cdot \xi_{\beta_{0}}\right)$. Since the conditions are invariant under the interchanging of $\alpha$ and $\widetilde{\alpha}$, we also put $y_{\widetilde{\alpha}}=x_{\widetilde{\alpha}} \cdot\left(\xi_{\beta} \cdot \xi_{\widetilde{\beta}_{0}}^{\prime}\right) /\left(\xi_{\beta}^{\prime} \cdot \xi_{\widetilde{\beta}_{0}}\right)$.

iv) There exist $\alpha^{\prime}, \gamma^{\prime} \in \Phi^{+}$and $\beta_{0} \in D$ such that $\alpha=\alpha_{0}$,

$$
\beta=\alpha^{\prime}+\gamma^{\prime}, \quad \beta_{0}=\alpha_{0}+\gamma^{\prime},
$$

and $\alpha=\alpha_{0}, \alpha^{\prime}$ are not singular to any other root in $D$. Then we let $y_{\alpha_{0}}=x_{\alpha_{0}} \cdot \xi_{\beta_{0}} / \xi_{\beta_{0}}^{\prime}$.

We check that the $y_{\alpha}$ are well defined. Suppose $\alpha \in \mathcal{A}$. If $\alpha \notin \operatorname{Supp}(x)$, then $y_{\alpha}=x_{\alpha}=0$; we assume $\alpha \in \operatorname{Supp}(x)$. If $\alpha$ is not singular to any root from $D$, then $y_{\alpha}=x_{\alpha}$. On the other hand, suppose $\alpha \in S(\beta)$ (i.e., $\beta=\alpha+\gamma, \gamma \in \Phi^{+}$). If $\gamma$ is not singular to any other root in $D$, then $f\left(\left[x, e_{\gamma}\right]\right)=\xi_{\beta} \cdot x_{\alpha} \cdot N_{\alpha \gamma} \neq 0$. This contradicts the choice of $x \in \mathfrak{b} \subset \mathfrak{a}=\mathfrak{r a d}_{\mathfrak{u}} f$; thus, there exists $\alpha_{0} \in \operatorname{Supp}(x)$ and $\beta_{0} \in D$ such that 
$\beta_{0} \neq \beta$ and $\beta_{0}=\alpha_{0}+\gamma$. Suppose there are $\widetilde{\beta}_{0} \in D, \gamma_{0} \in \Phi^{+}$such that $\widetilde{\beta}_{0} \neq \beta$ and $\widetilde{\beta}_{0}=\alpha+\gamma_{0}$. If $\widetilde{\beta}_{0}=\beta_{0}$, then

$$
\left(\beta_{0}, \beta\right)=\left(\beta_{0}, \alpha+\gamma\right)=\left(\widetilde{\beta}_{0}, \alpha\right)+\left(\beta_{0}, \gamma\right)=1 / 2+1 / 2=1,
$$

in contradiction with the orthogonality of $D$. Hence, $\widetilde{\beta}_{0} \neq \beta_{0}$.

If the root $\gamma_{0}$ is not singular to any root in $D$ except for $\widetilde{\beta}_{0}$, then $f\left(\left[x, e_{\gamma_{0}}\right]\right)=$ $\xi_{\widetilde{\beta}_{0}} \cdot x_{\alpha} \cdot N_{\alpha \gamma_{0}} \neq 0$, a contradiction. Therefore, there exists $\widehat{\beta} \neq \widetilde{\beta}_{0}$ such that $\widehat{\beta}=\widehat{\alpha}+\gamma_{0}$. If $\widehat{\beta} \neq \beta_{0}$, then we consider the set $\Psi=\left\langle\beta, \widehat{\beta}, \beta_{0}, \widetilde{\beta}_{0}, \alpha\right\rangle_{\mathbb{Z}} \cap \Phi$. Clearly, $\Psi$ is a root system of rank 5 , and $\gamma, \gamma_{0}, \alpha_{0}, \widehat{\alpha} \in \Psi$. Furthermore, $\Psi \cap \Phi^{+}$is a closed subset of $\Psi$; i.e., if $\zeta_{1}, \zeta_{2} \in \Psi \cap \Phi^{+}$and $\zeta_{1}+\zeta_{2} \in \Psi$, then $\zeta_{1}+\zeta_{2} \in \Psi \cap \Phi^{+}$. In accordance with [6. $\S 16$, Exercise 3], all roots in $\Psi \cap \Phi^{+}$are positive with respect to some subsystem of fundamental roots of $\Psi$. Thus, there is no loss of generality in assuming that $\beta, \widehat{\beta}, \beta_{0}$, $\widetilde{\beta}_{0}, \gamma, \gamma_{0}, \alpha, \alpha_{0}, \widetilde{\alpha}$ belong to $\Psi^{+}$.

Since the sum of two roots from different irreducible components of a root system is not a root, $\Psi$ is an irreducible simply laced root system. But since there are no four pairwise orthogonal roots in $A_{5}^{+}$, we have $\Psi \cong D_{5}$. Then the roots $\eta=\beta, \eta_{1}=\widetilde{\beta}_{0}$, $\eta_{2}=\beta_{0}, \eta_{3}=\widehat{\beta}, \theta=\gamma, \theta_{1}=\gamma_{0}, \psi=\alpha, \psi_{2}=\alpha_{0}$, and $\psi_{3}=\widehat{\alpha}$ form a nonadmissible subset of $D_{5}^{+}$of type 3. This contradicts Lemma 1.5.

We conclude that $\widehat{\beta}=\beta_{0}=\alpha_{0}+\gamma=\widehat{\alpha}+\gamma_{0}$. Denote $\widetilde{\alpha}=\widehat{\alpha}$, whence $\beta_{0}=\alpha_{0}+\gamma=$ $\widetilde{\alpha}+\gamma_{0}$. Then $\widetilde{\alpha}=\beta_{0}-\gamma_{0}=\beta_{0}-\left(\widetilde{\beta}_{0}-\alpha\right)=\beta_{0}-\widetilde{\beta}_{0}+\alpha$, so $(\beta, \widetilde{\alpha})=(\beta, \alpha)=1 / 2$. Consequently, $\widetilde{\alpha} \in S(\beta)$, because if $\beta \in S(\widetilde{\alpha})$, then $\beta<\beta_{0}$, and $\beta$ is not maximal among all roots in $D$. In other words, $\beta=\widetilde{\alpha}+\widetilde{\gamma}$ for some $\widetilde{\gamma} \in \Phi^{+}$. We see that

$$
\widetilde{\beta}_{0}=\alpha+\gamma_{0}=(\beta-\gamma)+\left(\beta_{0}-\widetilde{\alpha}\right)=\left(\beta_{0}-\gamma\right)+(\beta-\widetilde{\alpha})=\alpha_{0}+\widetilde{\gamma}
$$

Lemma 1.5 shows that $\alpha, \widetilde{\alpha}, \alpha_{0}, \gamma, \widetilde{\gamma}, \gamma_{0}$ are not orthogonal to any other $\operatorname{root} \beta_{2} \in D$. Indeed, suppose the contrary. Then the roots $\eta=\beta, \eta_{1}=\beta_{0}, \eta^{\prime}=\widetilde{\beta}_{0}, \theta=\alpha, \theta_{1}=\alpha_{0}$, $\theta_{1}^{\prime}=\widetilde{\alpha}, \psi=\gamma, \psi^{\prime}=\gamma_{0}, \psi_{1}^{\prime}=\widetilde{\gamma}$, and $\eta_{2}=\beta_{2}$ form a nonadmissible subset of $D_{5}^{+}$of type 4 . Thus, $\alpha$ is as in case iii), and the roots $\widetilde{\alpha}, \alpha_{0}, \beta_{0}, \widetilde{\beta}_{0}, \gamma, \widetilde{\gamma}, \gamma_{0}$ are determined uniquely.

Suppose now that $\beta=\alpha+\gamma, \beta_{0}=\alpha_{0}+\gamma$, but $\alpha$ is not singular to any other root in $D$ except for $\beta$. Suppose also that there exists $\widetilde{\gamma} \in \Phi^{+}$such that $\widetilde{\beta}_{0}=\alpha_{0}+\widetilde{\gamma}$. Then

$$
(\beta, \widetilde{\gamma})=\left(\beta, \widetilde{\beta}_{0}-\alpha_{0}\right)=\left(\beta, \widetilde{\beta}_{0}-\beta_{0}+\gamma\right)=1 / 2,
$$

because $\gamma \in S(\beta)$. This implies that $\widetilde{\gamma} \in S(\beta)$, because if $\beta \in S(\gamma)$, then $\beta<\beta_{0}$, and $\beta$ is not maximal. Let $\beta=\widetilde{\alpha}+\widetilde{\gamma}$ for some $\widetilde{\alpha} \in \Phi^{+}$. We note that $\widetilde{\alpha}$ is not singular to any other root in $D$ except for $\beta$. Indeed, otherwise the root $\widetilde{\alpha}$ is as in case iii). But this yields $\alpha \in S\left(\widetilde{\beta}_{0}\right)$, a contradiction.

Furthermore, $\gamma$ is not singular to any other root in $D$ except for $\beta$ and $\beta_{0}$. Indeed, if there exists $\beta_{2} \in D$ and $\alpha_{2} \in \Phi^{+}$such that $\beta_{2} \neq \beta, \beta_{2} \neq \beta_{0}$, and $\beta_{2}=\alpha_{2}+\gamma$, then the roots $\eta=\beta, \eta_{1}=\beta_{0}, \eta^{\prime}=\widetilde{\beta}_{0}, \eta_{2}=\beta_{2}, \theta=\alpha, \theta^{\prime}=\widetilde{\alpha}, \theta_{1}=\alpha_{0}, \theta_{2}=\alpha_{2}, \psi=\gamma$, and $\psi^{\prime}=\widetilde{\gamma}$ form a nonadmissible subset of $D_{5}^{+}$of type 1 . This contradicts Lemma 1.5. Similarly, $\widetilde{\gamma}$ is not singular to any other root in $D$ except for $\beta$ and $\widetilde{\beta}_{0}$. We see that $\alpha$ is as in case ii), and the roots $\beta_{0}, \widetilde{\beta}_{0}$ are determined uniquely.

Suppose now that $\beta=\alpha+\gamma, \beta_{0}=\alpha_{0}+\gamma$, and the roots $\alpha, \alpha_{0}$ are not singular to any other root in $D$ except for $\beta$ and $\beta_{0}$, respectively. Then $\alpha$ is as in case i), and the root $\beta_{0}$ is determined uniquely.

It remains to consider the case where $\alpha=\alpha_{0}$ is not singular to $\beta$, but there exists $\beta_{0} \in D$ and $\gamma^{\prime} \in \Phi^{+}$such that $\beta_{0} \neq \beta$ and $\beta_{0}=\alpha_{0}+\gamma^{\prime}$. Since $\alpha \notin S(\beta), \alpha$ does not 
belong to cases i)-iii). If $\gamma^{\prime} \notin S(\beta)$, then $\alpha=\alpha_{0}$ does not fall in case iv) either, so that $y_{\alpha_{0}}=x_{\alpha_{0}}$.

Now we suppose that $\gamma^{\prime} \in S(\beta)$, i.e., that there exists $\alpha^{\prime} \in \Phi^{+}$such that $\beta=\alpha^{\prime}+\gamma^{\prime}$. If $\alpha^{\prime} \in \operatorname{Supp}(x)$, then the root $\alpha^{\prime}$ is as in one of the cases i)-iii). This implies that $y_{\alpha_{0}}=x_{\alpha_{0}}$. If $\alpha^{\prime} \notin \operatorname{Supp}(x)$, then there exists $\beta_{1} \in D$ and $\alpha_{1} \in \Phi^{+}$such that $\beta \neq \beta$, $\beta_{1} \neq \beta_{0}$, and $\beta_{1}=\alpha_{1}+\gamma^{\prime}$ (if the converse is true, then $f\left(\left[x, e_{\gamma^{\prime}}\right]\right)=\xi_{\beta_{0}} \cdot x_{\alpha_{0}} \cdot N_{\alpha_{0} \gamma^{\prime}} \neq 0$, a contradiction). Hence, $\alpha^{\prime}, \alpha=\alpha_{0}$ are not singular to any other root in $D$ except for $\beta, \beta_{0}$, respectively.

Indeed, suppose there exists $\widetilde{\beta}_{0} \in D$ and $\widetilde{\gamma} \in \Phi^{+}$such that $\widetilde{\beta}_{0} \neq \beta_{0}$ and $\widetilde{\beta}_{0}=\alpha_{0}+\widetilde{\gamma}$. The root $\widetilde{\beta}_{0}$ does not coincide with $\beta$, because $\alpha=\alpha_{0}$ is not singular to $\beta$. If $\widetilde{\beta}_{0}$ coincides with $\beta_{1}$, then

$$
\left(\beta_{0}, \beta_{1}\right)=\left(\alpha_{0}, \gamma^{\prime}, \beta_{1}\right)=\left(\alpha_{0}, \widetilde{\beta}_{0}\right)+\left(\gamma^{\prime}, \beta_{1}\right)=1 / 2+1 / 2=1
$$

At the same time, $\widetilde{\gamma}=\widetilde{\beta}_{0}-\alpha_{0}=\widetilde{\beta}_{0}-\beta_{0}+\gamma^{\prime}$, so that $(\beta, \widetilde{\gamma})=1 / 2$ and $\widetilde{\gamma} \in S(\beta)$ (if $\beta \in S(\widetilde{\gamma})$, then $\beta<\beta_{0}$, whence $\beta$ is not maximal). However, if $\beta=\widetilde{\alpha}+\widetilde{\gamma}$ and $\widetilde{\alpha} \in \Phi^{+}$, then the roots $\eta=\beta, \eta_{1}=\beta_{0}, \eta_{2}=\beta_{1}, \eta^{\prime}=\widetilde{\beta}_{0}, \theta=\alpha^{\prime}, \theta_{1}=\alpha=\alpha_{0}, \theta_{2}=\alpha_{1}$, $\psi=\gamma^{\prime}$, and $\psi^{\prime}=\widetilde{\gamma}$ form a nonadmissible subset of $D_{5}^{+}$of type 1 , in contradiction with Lemma 1.5. This shows that $\widetilde{\beta}_{0} \neq \beta_{1}$.

On the other hand, suppose that there exists $\widetilde{\beta} \in D$ and $\widetilde{\gamma} \in \Phi^{+}$such that $\widetilde{\beta} \neq \beta$ and $\widetilde{\beta}=\alpha^{\prime}+\widetilde{\gamma}$. If $\widetilde{\beta}$ coincides with $\beta_{0}$, then $\left(\beta, \beta_{0}\right)=1$, whence $\widetilde{\beta} \neq \beta_{0}$; for the same reason, $\widetilde{\beta} \neq \beta_{1}$. It follows that the roots $\eta=\beta, \eta^{\prime}=\widetilde{\beta}, \eta_{1}=\beta_{0}, \eta_{2}=\beta_{1}, \theta=\gamma, \theta^{\prime}=\widetilde{\gamma}$, $\psi=\alpha^{\prime}, \psi_{1}=\alpha=\alpha_{0}$, and $\psi_{2}=\alpha_{1}$ form a nonadmissible subset of $D_{5}^{+}$of type 2 . This contradicts Lemma 1.5.

We have proved that if $\alpha=\alpha_{0} \notin S(\beta), \alpha \in S\left(\beta_{0}\right)$ for some $\beta_{0} \in D, \beta_{0} \neq \beta$, and $y_{\alpha} \neq x_{\alpha}$, then $\alpha=\alpha_{0}$ is as in case iv); in particular, $\beta_{0}$ is determined uniquely.

Therefore, if $y_{\alpha} \neq x_{\alpha}$, then $\alpha$ is as in one of the cases i) -iv), and the root $\beta_{0}$ is determined uniquely. Since the $y_{\alpha}$ depend only on $\beta_{0}$, they are well defined. Denote by $X, Y$ the $(|\mathcal{A}| \times l)$-matrices whose columns consist of the coordinates of the vectors $x_{1}$, $\ldots, x_{l} \in \mathfrak{b}$ and $y_{1}=\varphi\left(x_{1}\right), \ldots, y_{l}=\varphi\left(x_{l}\right)$ (respectively) in the basis $\left\{e_{\alpha}\right\}_{\alpha \in \mathcal{A}}$ of the algebra $\mathfrak{u}_{\mathcal{A}}$. Let $T$ be the diagonal $(|\mathcal{A}| \times|\mathcal{A}|)$-matrix whose $(\alpha, \alpha)$ th element equals

$$
t_{\alpha, \alpha}= \begin{cases}\xi_{\beta} / \xi_{\beta}^{\prime} & \text { if } \alpha \text { is as in case i), } \\ \left(\xi_{\beta} \cdot \xi_{\beta_{0}}^{\prime}\right) /\left(\xi_{\beta}^{\prime} \cdot \xi_{\beta_{0}}\right) & \text { if } \alpha \text { is as in either case ii) or case iii), } \\ \xi_{\beta_{0}} / \xi_{\beta_{0}}^{\prime} & \text { if } \alpha=\alpha_{0} \text { is as in case iv), } \\ 1 & \text { otherwise. }\end{cases}
$$

We see that $Y=T X$, but $\operatorname{det} T \neq 0$, whence $\operatorname{rk} X=\operatorname{rk} Y$. To conclude the proof, it remains to check that if $x \in \mathfrak{b}$, then $y \in \mathfrak{b}^{\prime}$, i.e., $f^{\prime}\left(\left[y, e_{\widehat{\gamma}}\right]\right)=0$ for all $\widehat{\gamma} \in \Phi^{+}$. We consider four cases.

1. First, suppose $(\beta, \widehat{\gamma})=0$, i.e., $\widehat{\gamma} \in \widetilde{\Phi}^{+}$and $\widehat{\gamma} \notin S(\beta)$. Let $\widehat{\gamma}$ be singular to the roots $\beta_{1}, \ldots, \beta_{l}$ in $\widetilde{D}=D \backslash\{\beta\}$ and not singular to any other root in $\widetilde{D}$. Denote $\alpha_{i}=\beta_{i}-\widehat{\gamma}$. Then $\left(\beta, \alpha_{i}\right)=\left(\beta, \beta_{i}-\widehat{\gamma}\right)=0$; therefore, $e_{\alpha_{i}}$ does not belong to $\operatorname{Supp}(x) \subset \mathcal{A}=\Phi^{+} \backslash \widetilde{\Phi}^{+}$. Thus, $f^{\prime}\left(\left[y, e_{\widehat{\gamma}}\right]\right)=0$.

2. Second, suppose $(\beta, \widehat{\gamma}) \neq 0$, i.e., $\widehat{\gamma} \in \mathcal{A}$. If $(\beta, \widehat{\gamma})=1$, then $\widehat{\gamma}=\beta$. But $\beta$ is not singular to any root in $D$, whence $f^{\prime}\left(\left[y, e_{\widehat{\gamma}}\right]\right)=f^{\prime}\left(\left[y, e_{\beta}\right]\right)=0$. If $(\beta, \widehat{\gamma})=-1 / 2$, then $\widehat{\gamma}$ is not singular to $\beta$; in this case, we denote $\gamma_{0}=\widehat{\gamma}$ (so, we must prove that $f^{\prime}\left(\left[y, e_{\gamma_{0}}\right]\right)=0$ ). If $\alpha=\widetilde{\beta}_{0}-\gamma_{0} \notin \operatorname{Supp}(x)$ for all $\widetilde{\beta}_{0} \in \widetilde{D}$ such that $\gamma_{0} \in S\left(\beta_{0}\right)$, then $f^{\prime}\left(\left[y, e_{\gamma_{0}}\right]\right)=0$. On the other hand, suppose there exists $\widetilde{\beta}_{0} \in \widetilde{D}$ and $\alpha \in \operatorname{Supp}(x)$ such that $\widetilde{\beta}_{0}=\alpha+\gamma_{0}$.

Since $(\beta, \alpha)=\left(\beta, \widetilde{\beta}_{0}-\gamma\right)=1 / 2$, we have $\alpha \in S(\beta)$ (if $\beta \in S(\alpha)$, then $\beta<\widetilde{\beta}_{0}$, so that $\beta$ is not maximal). In other words, there exists $\gamma \in \Phi^{+}$such that $\beta=\alpha+\gamma$. If $\gamma$ is 
not singular to any other root in $D$, then $f\left(\left[x, e_{\gamma}\right]\right)=\xi_{\beta} \cdot x_{\alpha} \cdot N_{\alpha \gamma} \neq 0$, a contradiction. Thus, there exists $\beta_{0} \in D$ with $\beta_{0} \neq \beta$ and $\alpha_{0} \in \Phi^{+}$such that $\beta_{0}=\alpha_{0}+\gamma$. Arguing as above, we see that $\alpha$ is as in case iii), and consequently,

$$
\begin{aligned}
f^{\prime}\left(\left[y, e_{\gamma_{0}}\right]\right) & =\xi_{\beta_{0}}^{\prime} \cdot N_{\widetilde{\alpha} \gamma_{0}} \cdot y_{\widetilde{\alpha}}+\xi_{\widetilde{\beta}_{0}}^{\prime} \cdot N_{\alpha \gamma_{0}} \cdot y_{\alpha} \\
& =\xi_{\beta_{0}}^{\prime} \cdot N_{\widetilde{\alpha} \gamma_{0}} \cdot x_{\widetilde{\alpha}} \cdot\left(\xi_{\beta} \cdot \xi_{\widetilde{\beta}_{0}}^{\prime}\right) /\left(\xi_{\beta}^{\prime} \cdot \xi_{\widetilde{\beta}_{0}}\right)+\xi_{\widetilde{\beta}_{0}}^{\prime} \cdot N_{\alpha \gamma_{0}} \cdot y_{\alpha} \cdot\left(\xi_{\beta} \cdot \xi_{\beta_{0}}^{\prime}\right) /\left(\xi_{\beta}^{\prime} \cdot \xi_{\beta_{0}}\right) \\
& =\left(\xi_{\beta_{0}} \cdot N_{\widetilde{\alpha} \gamma_{0}} \cdot x_{\widetilde{\alpha}}+\xi_{\widetilde{\beta}_{0}} \cdot N_{\alpha \gamma_{0}} \cdot x_{\alpha}\right) \cdot\left(\xi_{\beta_{0}}^{\prime} \cdot \xi_{\widetilde{\beta}_{0}}^{\prime} \cdot \xi_{\beta}\right) /\left(\xi_{\beta_{0}} \cdot \xi_{\widetilde{\beta}_{0}} \cdot \xi_{\beta}^{\prime}\right) \\
& =f\left(\left[x, e_{\gamma_{0}}\right]\right) \cdot\left(\xi_{\beta_{0}}^{\prime} \cdot \xi_{\widetilde{\beta}_{0}}^{\prime} \cdot \xi_{\beta}\right) /\left(\xi_{\beta_{0}} \cdot \xi_{\widetilde{\beta}_{0}} \cdot \xi_{\beta}^{\prime}\right)=0 .
\end{aligned}
$$

3. Third, suppose $(\beta, \widehat{\gamma})=1 / 2$ and $\widehat{\gamma} \in S(\beta)$; in this case, we denote $\gamma=\widehat{\gamma}$ (we must prove that $\left.f^{\prime}\left(\left[y, e_{\gamma}\right]\right)=0\right)$. Let $\beta=\alpha+\gamma, \alpha \in \Phi^{+}$. Also let $\gamma$ be singular to the roots $\beta_{1}$, $\ldots, \beta_{l}$ in $\widetilde{D}$ and not singular to any other root in $\widetilde{D}$. Denote $\alpha_{i}=\beta_{i}-\gamma$. If $\alpha \in \operatorname{Supp}(x)$, then, arguing as above, we conclude that $\alpha$ is as in one of the cases i)-iii). If $\alpha$ is as in case i), then all the roots $\alpha_{i}$ are as in case iv). Hence,

$$
\begin{aligned}
f^{\prime}\left(\left[y, e_{\gamma}\right]\right) & =\xi_{\beta}^{\prime} \cdot N_{\alpha \gamma} \cdot y_{\alpha}+\sum_{i=1}^{l} \xi_{\beta_{i}}^{\prime} \cdot N_{\alpha_{i} \gamma} \cdot y_{\alpha_{i}} \\
& =\xi_{\beta}^{\prime} \cdot N_{\alpha \gamma} \cdot x_{\alpha} \cdot \xi_{\beta} / \xi_{\beta}^{\prime}+\sum_{i=1}^{l} \xi_{\beta_{i}}^{\prime} \cdot N_{\alpha_{i} \gamma} \cdot x_{\alpha_{i}} \cdot \xi_{\beta_{i}} / \xi_{\beta_{i}}^{\prime} \\
& =\xi_{\beta} \cdot N_{\alpha \gamma} \cdot x_{\alpha}+\sum_{i=1}^{l} \xi_{\beta_{i}} \cdot N_{\alpha_{i} \gamma} \cdot x_{\alpha_{i}}=f\left(\left[x, e_{\gamma}\right]\right)=0 .
\end{aligned}
$$

On the other hand, if $\alpha$ is as in either case ii) or case iii), then $\gamma$ is not singular to any other root in $D$ except for $\beta$ and $\beta_{0}=\alpha_{0}+\gamma, y_{\alpha_{0}}=x_{\alpha_{0}}$, and

$$
\begin{aligned}
f^{\prime}\left(\left[y, e_{\gamma}\right]\right) & =\xi_{\beta}^{\prime} \cdot N_{\alpha \gamma} \cdot y_{\alpha}+\xi_{\beta_{0}}^{\prime} \cdot N_{\alpha_{0} \gamma} \cdot y_{\alpha_{0}} \\
& =\xi_{\beta}^{\prime} \cdot N_{\alpha \gamma} \cdot x_{\alpha} \cdot\left(\xi_{\beta} \cdot \xi_{\beta_{0}}^{\prime}\right) /\left(\xi_{\beta^{\prime}} \cdot \xi_{\beta_{0}}\right)+\xi_{\beta_{0}}^{\prime} \cdot N_{\alpha_{0} \gamma} \cdot x_{\alpha_{0}} \\
& =\left(\xi_{\beta} \cdot N_{\alpha \gamma} \cdot x_{\alpha}+\xi_{\beta_{0}} \cdot N_{\alpha_{0} \gamma} \cdot x_{\alpha_{0}}\right) \cdot \xi_{\beta_{0}}^{\prime} / \xi_{\beta_{0}} \\
& =f\left(\left[x, e_{\gamma}\right]\right) \cdot \xi_{\beta_{0}}^{\prime} / \xi_{\beta_{0}}=0 .
\end{aligned}
$$

Now assume that $\alpha \notin \operatorname{Supp}(x)$. If $\alpha_{i} \notin \operatorname{Supp}(x)$ for all $i$, then $f^{\prime}\left(\left[y, e_{\gamma}\right]\right)=0$ because $\operatorname{Supp}(x)=\operatorname{Supp}(y)$. At the same time, if $\alpha_{i} \in \operatorname{Supp}(x)$, then there exists $\alpha_{j}$ such that $i \neq j$ and $\alpha_{j} \in \operatorname{Supp}(x)$. We claim that the root $\alpha_{i}$ is not singular to any other root in $D$ except for $\beta_{i}$. Indeed, assume $\widetilde{\beta}_{i}=\alpha_{i}+\widetilde{\gamma}$ for some $\widetilde{\beta}_{i} \in D, \widetilde{\gamma} \in \Phi^{+}$. Since $\left(\beta, \alpha_{i}\right)=\left(\beta, \beta_{i}-\gamma\right)=-1 / 2$, we have $\alpha_{i} \notin S(\beta)$, whence $\widetilde{\beta}_{i} \neq \beta$. It follows that $(\beta, \widetilde{\gamma})=\left(\beta, \widetilde{\beta}_{i}-\alpha_{i}\right)=1 / 2$, so that $\widetilde{\gamma} \in S(\beta)$ (if $\beta \in S(\widetilde{\gamma})$, then $\beta<\widetilde{\beta}_{i}$ and $\beta$ is not maximal). Put $\beta=\widetilde{\alpha}+\widetilde{\gamma}, \widetilde{\alpha} \in \Phi^{+}$. If $\widetilde{\beta}_{i}=\beta_{j}$, then

$$
\left(\beta_{i}, \beta_{j}\right)=\left(\alpha_{i}+\gamma, \beta_{j}\right)=\left(\alpha_{i}, \widetilde{\beta}_{i}\right)+\left(\gamma, \beta_{j}\right)=1 / 2+1 / 2=1 .
$$

Hence, $\widetilde{\beta}_{i} \neq \beta_{j}$; thus, the roots $\eta=\beta, \eta_{1}=\beta_{i}, \eta_{2}=\beta_{j}, \eta^{\prime}=\widetilde{\beta}_{i}, \theta=\alpha, \theta^{\prime}=\widetilde{\alpha}, \theta_{1}=\alpha_{i}$, $\theta_{2}=\alpha_{j}, \psi=\gamma$, and $\psi^{\prime}=\widetilde{\gamma}$ form a nonadmissible subset of $D_{5}^{+}$of type 1 , in contradiction with Lemma 1.5. This shows that $\alpha_{i}$ is not singular to any other root in $D$ except for $\beta_{i}$, as required.

Similarly, suppose there exists $\widetilde{\beta} \in D$ and $\widetilde{\gamma} \in \Phi^{+}$such that $\widetilde{\beta} \neq \beta$ and $\widetilde{\beta}=\alpha+\widetilde{\gamma}$. If $\widetilde{\beta}$ coincides with $\beta_{i}$, then

$$
\left(\beta, \beta_{i}\right)=\left(\alpha+\gamma, \beta_{i}\right)=(\alpha, \widetilde{\beta})+\left(\gamma, \beta_{i}\right)=1 / 2+1 / 2=1 .
$$


Hence, $\widetilde{\beta} \neq \beta_{i}$. For the same reason, $\widetilde{\beta} \neq \beta_{j}$. But this shows that the $\operatorname{roots} \eta=\beta, \eta^{\prime}=\widetilde{\beta}$, $\eta_{1}=\beta_{i}, \eta_{2}=\beta_{j}, \theta=\gamma, \theta^{\prime}=\widetilde{\gamma}, \psi=\alpha, \psi_{1}=\alpha_{i}$, and $\psi_{2}=\alpha_{j}$ form a nonadmissible subset of $D_{5}^{+}$of type 2. This contradicts Lemma 1.5. We have proved that $\alpha$ and all $\alpha_{i} \in \operatorname{Supp}(x)$ are not singular to any other root in $D$ except for $\beta$ and $\beta_{i}$, respectively. This implies that all roots $\alpha_{i} \in \operatorname{Supp}(x)$ are as in case iv), whence

$$
\begin{aligned}
f^{\prime}\left(\left[y, e_{\gamma}\right]\right) & =\sum_{i=1}^{l} \xi_{\beta_{i}}^{\prime} \cdot N_{\alpha_{i} \gamma} \cdot y_{\alpha_{i}}=\sum_{i=1}^{l} \xi_{\beta_{i}}^{\prime} \cdot N_{\alpha_{i} \gamma} \cdot x_{\alpha_{i}} \cdot \xi_{\beta_{i}} / \xi_{\beta_{i}}^{\prime} \\
& =\sum_{i=1}^{l} \xi_{\beta_{i}} \cdot N_{\alpha_{i} \gamma} \cdot x_{\alpha_{i}}=f\left(\left[x, e_{\gamma}\right]\right)=0 .
\end{aligned}
$$

4. Finally, suppose $(\beta, \widehat{\gamma})=1 / 2$ and $\beta \in S(\widehat{\gamma})$. Then $\widehat{\gamma}$ is not singular to any root in $D$, which yields $f^{\prime}\left(\left[y, e_{\widehat{\gamma}}\right]\right)=0$. This concludes the proof of Proposition 2.5.

Remark 2.6. The case where $\Phi=A_{n}$ was considered by Panov in [16]. The case where $\Phi=D_{n}$ was considered by the author in [10]. Actually, a new result is only obtained for the root systems of types $E_{6}, E_{7}, E_{8}$. However, observe that the proofs are similar for all simply laced root systems.

\section{$\S 3$. Multiply laced Root systems}

3.1. Throughout the section, we assume $\Phi$ to be a reduced irreducible multiply laced root system (i.e., containing long and short roots). The cases of $B_{n}$ and $C_{n}$ were considered by the author in the paper [10]; now we assume that $\Phi$ is of type $F_{4}$ or $G_{2}$. First, suppose that $\Phi=G_{2}$ (this case is quite easy).

Recall that $G_{2}^{+}=\left\{\alpha_{1}, \alpha_{2}, \alpha_{1}+\alpha_{2}, 2 \alpha_{1}+\alpha_{2}, 2 \alpha_{1}+\alpha_{2}, 3 \alpha_{1}+2 \alpha_{2}\right\}$, where $\left\|\alpha_{1}\right\|^{2}=1$, $\left\|\alpha_{2}\right\|^{2}=3$ and the angle between the vectors $\alpha_{1}, \alpha_{2}$ equals $5 \pi / 6$. Let $D$ be an orthogonal subset of $\Phi^{+}$. Of course, $|D| \leq 2$. For $|D|=1$, there is nothing to prove, because $\Omega$ is an elementary orbit (see Example 1.7). There are three orthogonal subsets of $G_{2}^{+}$ of cardinality two; we shall consider all of them one by one. Note that $l(\sigma)=6$ and $l(\sigma)-s(\sigma)=6-2=4$, because $\sigma$ is the central symmetry. Denote $D=\left\{\beta_{1}, \beta_{2}\right\}$.

i) $\beta_{1}=\alpha_{1}, \beta_{2}=3 \alpha_{1}+2 \alpha_{2}$. Since the root $\alpha_{1}$ is fundamental, $S\left(\alpha_{1}\right)=\varnothing$. At the same time, the $\beta_{2}$-singular roots are the following: $S\left(\beta_{2}\right)=\left\{\alpha_{1}, 3 \alpha_{1}+\alpha_{2}\right\} \cup\left\{\alpha_{1}+\alpha_{2}, 2 \alpha_{1}+\alpha_{2}\right\}$. Put $\mathcal{M}=\left\{\alpha_{2}, \alpha_{1}+\alpha_{2}\right\}, \mathcal{P}=\Phi^{+} \backslash \mathcal{M}$ and $\mathfrak{p}=\sum_{\alpha \in \mathcal{P}} k e_{\alpha}$. It is easy to show that $\mathfrak{p} \subset \mathfrak{u}$ is an isotropic subspace, i.e., $f([x, y])=0$ for all $x, y \in \mathfrak{p}$. (Indeed, if $1 \leq i \leq 2$ and $\alpha, \gamma$ are $\beta_{i}$-singular, then $\mathcal{P}$ does not contain both of them.) Next, if $x \notin \mathfrak{p}$, then $\operatorname{Supp}(x)$ contains at least one of the roots $\gamma_{1}=\alpha_{2}$ and $\gamma_{2}=\alpha_{1}+\alpha_{2}$. Actually, if $x=x_{1} e_{\gamma_{1}}+\cdots$, $x_{1} \neq 0$, then $f\left(\left[x, e_{3 \alpha_{1}+\alpha_{2}}\right]\right)=\xi_{\beta_{2}} \cdot x_{1} \cdot N_{\gamma_{1}, 3 \alpha_{1}+\alpha_{2}} \neq 0$. Similarly, if $x=x_{2} e_{\gamma_{2}}+\cdots$, $x_{2} \neq 0$, then $f\left(\left[x, e_{2 \alpha_{1}+\alpha_{2}}\right]\right)=\xi_{\beta_{2}} \cdot x_{2} \cdot N_{\gamma_{2}, 2 \alpha_{1}+\alpha_{2}} \neq 0$. Thus, $\mathfrak{p}$ is a maximal isotropic subspace with respect to the inclusion order. Hence, $\operatorname{dim} \Omega$ does not depend on $\xi$ and equals $2 \cdot \operatorname{codim}_{\mathfrak{u}} \mathfrak{p}=4=l(\sigma)-s(\sigma)$ (see, e.g., [2, §3]).

ii) $\beta_{1}=\alpha_{1}+\alpha_{2}, \beta_{2}=3 \alpha_{1}+\alpha_{2}$. Here $S\left(\beta_{1}\right)=\left\{\alpha_{1}, \alpha_{2}\right\}, S\left(\beta_{2}\right)=\left\{\alpha_{1}, 2 \alpha_{1}+\alpha_{2}\right\}$. Put $\mathcal{M}=\left\{\alpha_{1}\right\}$. Let $\mathcal{P}, \mathfrak{p}$ be defined as above. Evidently, $\mathfrak{p}$ is an isotropic subspace. On the other hand, if $x=x_{1} e_{\alpha_{1}}+\cdots \notin \mathfrak{p}$, then $f\left(\left[x, e_{\alpha_{2}}\right]\right)=\xi_{\beta_{1}} \cdot x_{1} \cdot N_{\alpha_{1} \alpha_{2}} \neq 0$, whence $\mathfrak{p}$ is a maximal isotropic subspace. Consequently, $\operatorname{dim} \Omega$ does not depend on $\xi$ and equals $2 \cdot \operatorname{codim}_{\mathfrak{u}} \mathfrak{p}=2<4=l(\sigma)-s(\sigma)$.

iii) $\beta_{1}=\alpha_{2}, \beta_{2}=2 \alpha_{1}+\alpha_{2}$. Since the root $\alpha_{2}$ is fundamental, $S\left(\alpha_{2}\right)=\varnothing$. At the same time, $S\left(\beta_{2}\right)=\left\{\alpha_{1}, \alpha_{1}+\alpha_{2}\right\}$. Putting $\mathcal{M}=\left\{\alpha_{1}\right\}$, we see that $\operatorname{dim} \Omega$ does not depend on $\xi$ and equals $2<4=l(\sigma)-s(\sigma)$, as at the preceding step.

3.2. Now we consider the more complicated case where $\Phi=F_{4}$. Recall that

$$
F_{4}^{+}=\left\{\varepsilon_{i}, \varepsilon_{i} \pm \varepsilon_{j},\left(\varepsilon_{1} \pm \varepsilon_{2} \pm \varepsilon_{3} \pm \varepsilon_{4}\right) / 2,1 \leq i<j \leq 4\right\} \quad \text { (the signs are independent) }
$$


where $\alpha_{1}=\varepsilon_{2}-\varepsilon_{3}, \alpha_{2}=\varepsilon_{3}-\varepsilon_{4}, \alpha_{3}=\varepsilon_{4}$, and $\alpha_{4}=\left(\varepsilon_{1}-\varepsilon_{2}-\varepsilon_{3}-\varepsilon_{4}\right) / 2$ are fundamental roots (here $\left\{\varepsilon_{i}\right\}_{i=1}^{4}$ is the standard basis of $\mathbb{R}^{4}$ ). For convenience, put $\widetilde{\Phi}^{+}=\left\{\varepsilon_{i}, \varepsilon_{i} \pm \varepsilon_{j}, 1 \leq i<j \leq 4\right\}$ and $\mathcal{B}=\Phi^{+} \backslash \widetilde{\Phi}^{+}$. We have $\widetilde{\Phi} \cong B_{4}$ as root systems, where $\widetilde{\Phi}= \pm \widetilde{\Phi}^{+}$.

We begin with the case where $D \subset \widetilde{\Phi}^{+}$. Let $\widetilde{W}$ denote the Weyl group of the root system $\widetilde{\Phi}$. We also denote by $\widetilde{\sigma}$ the involution in $\widetilde{W}$ corresponding to the subset $D$. Clearly, $s(\widetilde{\sigma})=s(\sigma)=|D|$ and $l(\widetilde{\sigma}) \leq l(\sigma)$. Precisely, $\mathcal{F}=\widetilde{\mathcal{F}}+\#\{\alpha \in \mathcal{B} \mid \sigma \alpha<0\}$ (here we put $\mathcal{F}=l(\sigma)-s(\sigma)$ and $\widetilde{\mathcal{F}}=l(\widetilde{\sigma})-s(\widetilde{\sigma}))$.

As above, we denote $\widetilde{\mathfrak{u}}=\sum_{\alpha \in \widetilde{\Phi}^{+}} k e_{\alpha}$ and $\mathfrak{u}_{\mathcal{B}}=\sum_{\alpha \in \mathcal{B}} k e_{\alpha}$ (hence, $\mathfrak{u}=\widetilde{\mathfrak{u}} \oplus \mathfrak{u}_{\mathcal{B}}$ as vector spaces) and set $\widetilde{f}=\left.f\right|_{\mathfrak{u}}, \widetilde{U}=\exp (\widetilde{\mathfrak{u}})$. Let $\widetilde{\Omega} \subset \widetilde{\mathfrak{u}}^{*}$ be the orbit of $\widetilde{f}$ under the coadjoint action of the group $\widetilde{U}$. Put $\mathfrak{a}=\mathfrak{r a d}_{\mathfrak{u}} f$ and $\widetilde{\mathfrak{a}}=\mathfrak{r} \mathfrak{a} \mathfrak{d}_{\mathfrak{u}} \tilde{f}$. From [10, Theorem 1.2] it follows that $\operatorname{dim} \widetilde{\Omega}=\widetilde{\mathcal{F}}-\vartheta$, where $\vartheta$ depends only on $D$ but not on $\xi$. Finally, put $\mathfrak{b}=\mathfrak{a} \cap \mathfrak{u}_{\mathcal{B}}$.

Lemma 3.1. We have $\mathfrak{a}=\widetilde{\mathfrak{a}} \oplus \mathfrak{b}$ as vector spaces (cf. Lemma 2.2).

Proof. Suppose $x \in \widetilde{\mathfrak{a}}, \alpha \in \operatorname{Supp}(x)$. If $\gamma \in \widetilde{\Phi}$, then $f\left(\left[x, e_{\gamma}\right]\right)=0$ because $\widetilde{\mathfrak{a}}=\mathfrak{r a d} \mathfrak{d}_{\mathfrak{u}} f$ is the radical of $f$. If $\gamma \in \mathcal{B}$, then $\alpha+\gamma \in \mathcal{B}$ because $f\left(\left[e_{\alpha}, e_{\gamma}\right]\right)=0$. We see that $f\left(\left[x, e_{\gamma}\right]\right)=0$ for all $\gamma \in \Phi^{+}$, whence $x \in \mathfrak{a}$. Thus, $\tilde{\mathfrak{a}} \subset \mathfrak{a}$. On the other hand, suppose $x=y+z \in \mathfrak{a}, y \in \widetilde{\mathfrak{u}}, z \in \mathfrak{u}_{\mathcal{B}}, \alpha \in \operatorname{Supp}(z)$, and $\gamma \in \widetilde{\Phi}^{+}$. Then $\alpha+\gamma \in \mathcal{B}$, so that $f\left(\left[z, e_{\gamma}\right]\right)=0$ and $f\left(\left[y, e_{\gamma}\right]\right)=0$, i.e., $y \in \tilde{\mathfrak{a}} \subset \mathfrak{a}$. Hence, $z \in \mathfrak{a}$ and $\mathfrak{a}=\widetilde{\mathfrak{a}}+\mathfrak{b}$. But $\widetilde{\mathfrak{a}} \cap \mathfrak{b}=0$. The proof is complete.

Lemma 3.2. We have

$$
\mathfrak{b}=\left\langle e_{\alpha}, \alpha \in \mathcal{B} \mid \sigma \alpha>0\right\rangle_{k}
$$

(cf. Lemma 2.3).

Proof. Set $\widetilde{\mathcal{B}}=\{\alpha \in \mathcal{B} \mid \sigma \alpha>0\}$. First, suppose that $D$ does not contain the roots $\varepsilon_{1}$, $\varepsilon_{1} \pm \varepsilon_{j}, j=2,3,4$. Then $\sigma \alpha=\varepsilon_{1} / 2 \pm \cdots>0$ for all $\alpha \in \mathcal{B}$, whence $\widetilde{\mathcal{B}}=\mathcal{B}$. In this case, $\mathcal{B} \cap \bigcup_{\beta^{\prime} \in D} S\left(\beta^{\prime}\right)=\varnothing$. Hence, $\mathfrak{b}=\mathfrak{u}_{\mathcal{B}}$, as required.

Second, suppose $\beta=\varepsilon_{1} \in D$. Then $D$ does not contain the roots $\varepsilon_{1} \pm \varepsilon_{j}, j=2,3,4$, so that $\sigma \alpha=-\varepsilon_{1} / 2 \pm \cdots<0$ for all $\alpha \in \mathcal{B}$. This implies that $\widetilde{\mathcal{B}}=\varnothing$. On the other hand, if $\alpha \in \mathcal{B}$, then $\gamma=\beta-\alpha$ is not singular to any other root in $D$ except for $\beta$. Therefore, $f\left(\left[x, e_{\gamma}\right]\right)=\xi_{\beta} \cdot x_{\alpha} \cdot N_{\alpha \gamma} \neq 0$ if $x=x_{\alpha} e_{\alpha}+\cdots \in \mathfrak{u}_{\mathcal{B}}$. Thus, $\mathfrak{b}=0$, as required.

Third, suppose there exists $j$ such that $\beta=\varepsilon_{1}-\varepsilon_{j} \in D$ and $\varepsilon_{1}+\varepsilon_{j} \notin D$. In this case, $\sigma \alpha>0$ if and only if $\alpha=\left(\varepsilon_{1}+\varepsilon_{j} \pm \cdots\right) / 2$. If $\gamma \in \widetilde{\Phi}^{+}$, then $\alpha+\gamma \in \mathcal{B}$, whence $f\left(\left[e_{\alpha}, e_{\gamma}\right]\right)=0$. At the same time, if $\gamma \in \mathcal{B}$, then the coefficient of $\varepsilon_{j}$ in $\gamma$ is at least $-1 / 2$, so that $\alpha+\gamma \neq \beta$. On the other hand, since $\alpha+\gamma=\varepsilon_{1} \pm \cdots$, we have $\alpha+\gamma \notin S(\beta)$ for all $\beta \in D$. This shows that $e_{\alpha} \in \mathfrak{b}$. But if $x \in \mathfrak{u}_{\mathcal{B}}$ and $\alpha=\left(\varepsilon_{1}-\varepsilon_{j} \pm \cdots\right) / 2 \in \operatorname{Supp}(x)$, then $\gamma=\beta-\alpha$ is not singular to any other root in $D$ except for $\beta$. If $x=x_{\alpha} e_{\alpha}+\cdots$, then $f\left(\left[x, e_{\gamma}\right]\right)=\xi_{\beta} \cdot x_{\alpha} \cdot N_{\alpha \gamma} \neq 0$, a contradiction. Thus, $\mathfrak{b}=\left\langle e_{\alpha}, \alpha \in \widetilde{\mathcal{B}}\right\rangle_{k}$.

Similarly, if there exists $j$ such that $\beta=\varepsilon_{1}+\varepsilon_{j} \in D$ and $\varepsilon_{1}-\varepsilon_{j} \notin D$, then $\sigma \alpha>0$ if and only if $\alpha=\left(\varepsilon_{1}-\varepsilon_{j} \pm \cdots\right) / 2$, i.e., $e_{\alpha} \in \mathfrak{b}$. At the same time, if $\alpha=\left(\varepsilon_{1}+\varepsilon_{j} \pm \cdots\right)$, then $\gamma=\beta-\alpha$ is not singular to any other root in $D$ except for $\beta$, whence $f\left(\left[x, e_{\gamma}\right]\right) \neq 0$. It follows that if $x \in \mathfrak{u}_{\mathcal{B}}$ and $\alpha \in \operatorname{Supp}(x)$, then $x \notin \mathfrak{a}$. Hence, $\mathfrak{b}=\left\langle e_{\alpha}, \alpha \in \widetilde{\mathcal{B}}\right\rangle_{k}$.

Finally, suppose $\varepsilon_{1}-\varepsilon_{j}, \varepsilon_{1}+\varepsilon_{j} \in D$ for some $j$. Then $\sigma \alpha=-\varepsilon_{1} / 2 \pm \cdots<0$ for all $\alpha \in \mathcal{B}$, so that $\widetilde{\mathcal{B}}=\varnothing$. Let $\alpha$ be a root in $\mathcal{B}$. Then $\alpha=\left(\varepsilon_{1}+z \cdot \varepsilon_{j} \pm \cdots\right) / 2, z= \pm 1$; therefore, $\alpha$ is not singular to any other root in $D$ except for $\beta=\varepsilon_{1}+z \cdot \varepsilon_{j}$; this is also true for the root $\gamma=\beta-\alpha$. Arguing as above, we see that $x \notin \mathfrak{a}$ if $x \in \mathfrak{u}_{\mathcal{B}}$ and $\alpha \in \operatorname{Supp}(x)$. Thus, $\mathfrak{b}=0$, as required. The proof is complete. 
The two preceding lemmas show that

$$
\begin{aligned}
\operatorname{dim} \Omega & =\operatorname{codim}_{\mathfrak{u}} \mathfrak{a}=\operatorname{dim} \mathfrak{u}-\operatorname{dim} \mathfrak{a}=\left|\Phi^{+}\right|-(\operatorname{dim} \widetilde{\mathfrak{a}}+\operatorname{dim} \mathfrak{b}) \\
& =\left|\widetilde{\Phi}^{+}\right|+|\mathcal{B}|-\operatorname{dim} \widetilde{\mathfrak{a}}-\operatorname{dim} \mathfrak{b}=\left(\left|\widetilde{\Phi}^{+}\right|-\operatorname{dim} \widetilde{\mathfrak{a}}\right)+|\mathcal{B}|-\operatorname{dim} \mathfrak{b} \\
& =(\operatorname{dim} \widetilde{\mathfrak{u}}-\operatorname{dim} \widetilde{\mathfrak{a}})+|\mathcal{B}|-\#\{\alpha \in \mathcal{B} \mid \sigma \alpha>0\} \\
& =\operatorname{codim}_{\widetilde{\mathfrak{u}}} \widetilde{\mathfrak{a}}+\#\{\alpha \in \mathcal{B} \mid \sigma \alpha<0\} \\
& =\operatorname{dim} \widetilde{\Omega}+\mathcal{F}-\widetilde{\mathcal{F}}=\widetilde{\mathcal{F}}-\vartheta+\mathcal{F}-\widetilde{\mathcal{F}}=\mathcal{F}-\vartheta .
\end{aligned}
$$

Therefore, $\operatorname{dim} \Omega$ does not depend on $\xi$ and does not exceed $\mathcal{F}$. In other words, Theorem 0.2 holds true for all orthogonal subsets of $\widetilde{\Phi}^{+}$.

3.3. In this subsection, we consider orthogonal subsets of $F_{4}^{+}$that are not contained in $\widetilde{\Phi}^{+}$. In other words, we assume that the intersection of $D$ with $\mathcal{B}$ is nonempty. It is easy to show that if $\beta_{1}, \beta_{2} \in \mathcal{B}$ are orthogonal and $\beta_{1} \notin S\left(\beta_{2}\right)$, then $\beta_{2} \in S\left(\beta_{1}\right)$, so that there is no loss of generality in assuming that $|D \cap \mathcal{B}|=1$ (see Lemma 1.3). Clearly, $D$ does not contain the roots $\varepsilon_{i}, 1 \leq i \leq 4$; in other words, there exists a unique short root contained in $D$.

Since the root system $F_{4}$ is self-dual, there exists a bijection $\varphi: F_{4} \rightarrow F_{4}$ such that $\varphi\left(F_{4}^{+}\right)=F_{4}^{+}$and $\varphi(S(\alpha))=S(\varphi(\alpha))$ for a given positive root $\alpha$. Furthermore,

$$
\varphi(\mathcal{B})=\left\{\varepsilon_{1} \pm \varepsilon_{j}, \varepsilon_{2} \pm \varepsilon_{j}, j=3,4\right\} \quad \text { (the signs are independent) }
$$

and $\varphi(\widetilde{\Phi}) \cong C_{4}$. So, if $D \subset \varphi\left(\widetilde{\Phi}^{+}\right)$, then the results of [10] can be applied, and Theorem 0.2 is valid for the subset $D$.

Let

$$
\beta=\left(\varepsilon_{1}+z_{2} \cdot \varepsilon_{2}+z_{3} \cdot \varepsilon_{3}+z_{4} \cdot \varepsilon_{4}\right) / 2 \in D, \quad z_{j}= \pm 1 .
$$

By the previous remarks, $\widetilde{D}=D \backslash\{\beta\}$ coincides either with one of the subsets $\left\{\varepsilon_{1}-z_{3} \cdot \varepsilon_{3}\right.$, $\left.\varepsilon_{2}-z_{2} z_{4} \cdot \varepsilon_{4}\right\}$ and $\left\{\varepsilon_{1}-z_{4} \cdot \varepsilon_{3}, \varepsilon_{2}-z_{2} z_{3} \cdot \varepsilon_{3}\right\}$, or with a one-element subset of them. Since the $\operatorname{root} \beta=\left(\varepsilon_{1}-\varepsilon_{2}-\varepsilon_{3}-\varepsilon_{4}\right) / 2$ is fundamental, $\Omega=\xi_{\beta} e_{\beta}^{*}+\widetilde{\Omega}$, whence $\operatorname{dim} \Omega=\operatorname{dim} \widetilde{\Omega}$, where $\widetilde{\Omega}=\Omega_{\widetilde{D}, \widetilde{\xi}} \subset \mathfrak{u}^{*}, \widetilde{\xi}=\xi \backslash\left\{\xi_{\beta}\right\}$. On the other hand, on the set of positive roots nonequal to $\beta, r_{\beta}$ acts by permutations, so that

$$
l(\sigma)-s(\sigma)=(l(\widetilde{\sigma})+1)-(s(\widetilde{\sigma})+1)=l(\widetilde{\sigma})-s(\widetilde{\sigma}),
$$

where $\widetilde{\sigma}$ is the involution in $W$ corresponding to $\widetilde{D}$. Since Theorem 0.2 holds true for the orbit $\widetilde{\Omega}$, we may assume that $\beta \neq\left(\varepsilon_{1}-\varepsilon_{2}-\varepsilon_{3}-\varepsilon_{4}\right) / 2$. By the same argument, it can be assumed that the fundamental root $\alpha_{1}=\varepsilon_{2}-\varepsilon_{3}$ does not belong to $D$ (if $|D|=2$, then the problem reduces to elementary orbits; if $|D|=3$, then the problem reduces to orbits associated with two-element subsets).

For a given $D$, denote by $\mathcal{M} \subset \Phi^{+}$a subset satisfying the following conditions. First, if $\alpha+\gamma=\beta \in D$, then $|\mathcal{M} \cap\{\alpha, \gamma\}|=1$. Second, for a given $\gamma \in \mathcal{M}$, there exists $\alpha \in \mathcal{P}$ such that $\alpha+\gamma=\beta \in D$ (here $\left.\mathcal{P}=\Phi^{+} \backslash \mathcal{M}\right)$. Third, $(\alpha+\mathcal{M}) \cap D$ consists either of the root $\beta$, or of the roots $\beta, \widetilde{\beta}=\alpha+\widetilde{\gamma}, \widetilde{\gamma} \in \mathcal{M}$, and in the latter case $\widetilde{\gamma} \in S(\beta),(\widetilde{\alpha}+\mathcal{M}) \cap D=\{\beta\}$, where $\widetilde{\alpha}=\beta-\widetilde{\gamma} \in \mathcal{P}$. Assume $\mathcal{M}$ exists. Then $\mathfrak{p}=\sum_{\alpha \in \mathcal{P}} k e_{\alpha}$ is a maximal isotropic subspace of the canonical form on $\Omega$; it follows that $\operatorname{dim} \Omega=2 \cdot \operatorname{codim}_{\mathfrak{u}} \mathfrak{p}=2 \cdot|\mathcal{M}|$ does not depend on $\xi$.

In the table that follows we list subsets $\mathcal{M}$ for all remaining $D$ (the signs \pm in the table are independent). It is straightforward to check that they satisfy the above conditions. We also compute the numbers $\mathcal{F}=l(\sigma)-s(\sigma)$ for all $D$. It is easily seen that $2 \cdot|\mathcal{M}| \leq \mathcal{F}$ for all $D$. This concludes the proof of Theorem 0.2 . 


\begin{tabular}{|c|c|c|c|}
\hline Subset $D$ & Subset $\mathcal{M}$ & $|\mathcal{M}|$ & $\mathcal{F}$ \\
\hline $\begin{array}{l}\text { 1) } \varepsilon_{1}+\varepsilon_{3} \\
\left(\varepsilon_{1}-\varepsilon_{2}-\varepsilon_{3}+\varepsilon_{4}\right) / 2\end{array}$ & $\begin{array}{l}\varepsilon_{1}, \varepsilon_{4}, \varepsilon_{1}-\varepsilon_{2}, \varepsilon_{1} \pm \varepsilon_{4} \\
\left(\varepsilon_{1}+\varepsilon_{2}+\varepsilon_{3} \pm \varepsilon_{4}\right) / 2\end{array}$ & 7 & 14 \\
\hline $\begin{array}{l}\text { 2) } \varepsilon_{1}-\varepsilon_{4} \\
\left(\varepsilon_{1}-\varepsilon_{2}-\varepsilon_{3}+\varepsilon_{4}\right) / 2\end{array}$ & $\begin{array}{l}\varepsilon_{1}-\varepsilon_{2}, \varepsilon_{1}-\varepsilon_{3} \\
\left(\varepsilon_{1} \pm \varepsilon_{2}-\varepsilon_{3}-\varepsilon_{4}\right) / 2\end{array}$ & 4 & 8 \\
\hline $\begin{array}{l}\text { 3) } \varepsilon_{2}+\varepsilon_{4} \\
\left(\varepsilon_{1}-\varepsilon_{2}-\varepsilon_{3}+\varepsilon_{4}\right) / 2\end{array}$ & $\varepsilon_{4}, \varepsilon_{2}-\varepsilon_{3}$ & 2 & 4 \\
\hline $\begin{array}{l}\text { 4) } \varepsilon_{1}-\varepsilon_{3} \\
\left(\varepsilon_{1}-\varepsilon_{2}+\varepsilon_{3}-\varepsilon_{4}\right) / 2\end{array}$ & $\begin{array}{l}\varepsilon_{1}-\varepsilon_{2} \\
\left(\varepsilon_{1}-\varepsilon_{2}-\varepsilon_{3} \pm \varepsilon_{4}\right) / 2\end{array}$ & 3 & 6 \\
\hline $\begin{array}{l}\text { 5) } \varepsilon_{1}+\varepsilon_{4}, \\
\left(\varepsilon_{1}-\varepsilon_{2}+\varepsilon_{3}-\varepsilon_{4}\right) / 2\end{array}$ & $\begin{array}{l}\varepsilon_{1}, \varepsilon_{3}, \varepsilon_{1}-\varepsilon_{2}, \varepsilon_{1}-\varepsilon_{3}, \\
\left(\varepsilon_{1}-\varepsilon_{2} \pm \varepsilon_{3}+\varepsilon_{4}\right) / 2\end{array}$ & 6 & 12 \\
\hline $\begin{array}{l}\text { 6) } \varepsilon_{2}+\varepsilon_{3} \\
\left(\varepsilon_{1}-\varepsilon_{2}+\varepsilon_{3}-\varepsilon_{4}\right) / 2\end{array}$ & $\varepsilon_{3}, \varepsilon_{3} \pm \varepsilon_{4}$ & 3 & 6 \\
\hline $\begin{array}{l}\text { 7) } \varepsilon_{2}-\varepsilon_{4}, \\
\left(\varepsilon_{1}-\varepsilon_{2}+\varepsilon_{3}-\varepsilon_{4}\right) / 2\end{array}$ & $\begin{array}{l}\varepsilon_{3}-\varepsilon_{4}, \\
\left(\varepsilon_{1}-\varepsilon_{2}-\varepsilon_{3}-\varepsilon_{4}\right) / 2\end{array}$ & 2 & 4 \\
\hline $\begin{array}{l}\text { 8) } \varepsilon_{1}-\varepsilon_{3} \\
\left(\varepsilon_{1}-\varepsilon_{2}+\varepsilon_{3}+\varepsilon_{4}\right) / 2\end{array}$ & $\begin{array}{l}\varepsilon_{4}, \varepsilon_{1}-\varepsilon_{2} \\
\left(\varepsilon_{1}-\varepsilon_{2}-\varepsilon_{3} \pm \varepsilon_{4}\right) / 2\end{array}$ & 4 & 8 \\
\hline $\begin{array}{l}\text { 9) } \varepsilon_{1}-\varepsilon_{4} \\
\left(\varepsilon_{1}-\varepsilon_{2}+\varepsilon_{3}+\varepsilon_{4}\right) / 2\end{array}$ & $\begin{array}{l}\varepsilon_{3}, \varepsilon_{1}-\varepsilon_{2}, \varepsilon_{1}-\varepsilon_{3} \\
\left(\varepsilon_{1}-\varepsilon_{2} \pm \varepsilon_{3}-\varepsilon_{4}\right) / 2\end{array}$ & 5 & 10 \\
\hline $\begin{array}{l}\text { 10) } \varepsilon_{2}+\varepsilon_{3} \\
\left(\varepsilon_{1}-\varepsilon_{2}+\varepsilon_{3}+\varepsilon_{4}\right) / 2\end{array}$ & $\begin{array}{l}\varepsilon_{3}, \varepsilon_{3} \pm \varepsilon_{4} \\
\left(\varepsilon_{1}-\varepsilon_{2}+\varepsilon_{3}-\varepsilon_{4}\right) / 2\end{array}$ & 4 & 8 \\
\hline $\begin{array}{l}\text { 11) } \varepsilon_{2}+\varepsilon_{4} \\
\left(\varepsilon_{1}-\varepsilon_{2}+\varepsilon_{3}+\varepsilon_{4}\right) / 2\end{array}$ & $\begin{array}{l}\varepsilon_{4}, \varepsilon_{3}+\varepsilon_{4} \\
\left(\varepsilon_{1}-\varepsilon_{2}-\varepsilon_{3}+\varepsilon_{4}\right) / 2\end{array}$ & 3 & 6 \\
\hline $\begin{array}{l}\text { 12) } \varepsilon_{1}+\varepsilon_{3} \\
\left(\varepsilon_{1}+\varepsilon_{2}-\varepsilon_{3}-\varepsilon_{4}\right) / 2\end{array}$ & $\begin{array}{l}\varepsilon_{1}, \varepsilon_{2}+\varepsilon_{3}, \varepsilon_{1} \pm \varepsilon_{4} \\
\left(\varepsilon_{1}-\varepsilon_{2} \pm \varepsilon_{3} \pm \varepsilon_{4}\right) / 2\end{array}$ & 8 & 16 \\
\hline $\begin{array}{l}\text { 13) } \varepsilon_{1}+\varepsilon_{4} \\
\left(\varepsilon_{1}+\varepsilon_{2}-\varepsilon_{3}-\varepsilon_{4}\right) / 2\end{array}$ & $\begin{array}{l}\varepsilon_{1}, \varepsilon_{1}-\varepsilon_{2}, \varepsilon_{1}-\varepsilon_{3} \\
\left(\varepsilon_{1}-\varepsilon_{2} \pm \varepsilon_{3} \pm \varepsilon_{4}\right) / 2\end{array}$ & 7 & 14 \\
\hline $\begin{array}{l}\text { 14) } \varepsilon_{2}+\varepsilon_{3} \\
\left(\varepsilon_{1}+\varepsilon_{2}-\varepsilon_{3}-\varepsilon_{4}\right) / 2\end{array}$ & $\varepsilon_{2}, \varepsilon_{2}-\varepsilon_{3}, \varepsilon_{2} \pm \varepsilon_{4}$ & 4 & 8 \\
\hline $\begin{array}{l}\text { 15) } \varepsilon_{2}+\varepsilon_{4}, \\
\left(\varepsilon_{1}+\varepsilon_{2}-\varepsilon_{3}-\varepsilon_{4}\right) / 2\end{array}$ & $\varepsilon_{2}, \varepsilon_{2}-\varepsilon_{3}, \varepsilon_{2}-\varepsilon_{4}$ & 3 & 6 \\
\hline $\begin{array}{l}\text { 16) } \varepsilon_{1}+\varepsilon_{3} \\
\left(\varepsilon_{1}+\varepsilon_{2}-\varepsilon_{3}+\varepsilon_{4}\right) / 2\end{array}$ & $\begin{array}{l}\varepsilon_{1}, \varepsilon_{4}, \varepsilon_{1}-\varepsilon_{2}, \varepsilon_{1} \pm \varepsilon_{4} \\
\left(\varepsilon_{1}-\varepsilon_{2} \pm \varepsilon_{3} \pm \varepsilon_{4}\right) / 2\end{array}$ & 9 & 18 \\
\hline $\begin{array}{l}\text { 17) } \varepsilon_{1}-\varepsilon_{4} \\
\left(\varepsilon_{1}+\varepsilon_{2}-\varepsilon_{3}+\varepsilon_{4}\right) / 2\end{array}$ & $\begin{array}{l}\varepsilon_{2}, \varepsilon_{1}-\varepsilon_{2}, \varepsilon_{1}-\varepsilon_{3} \\
\varepsilon_{2}-\varepsilon_{3},\left(\varepsilon_{1} \pm \varepsilon_{2}-\varepsilon_{3}-\varepsilon_{4}\right) / 2\end{array}$ & 6 & 12 \\
\hline $\begin{array}{l}\text { 18) } \varepsilon_{2}+\varepsilon_{3} \\
\left(\varepsilon_{1}+\varepsilon_{2}-\varepsilon_{3}+\varepsilon_{4}\right) / 2\end{array}$ & $\begin{array}{l}\varepsilon_{2}, \varepsilon_{2} \pm \varepsilon_{4} \\
\varepsilon_{4}, \varepsilon_{2}-\varepsilon_{3}\end{array}$ & 5 & 10 \\
\hline $\begin{array}{l}\text { 19) } \varepsilon_{2}-\varepsilon_{4} \\
\left(\varepsilon_{1}+\varepsilon_{2}-\varepsilon_{3}+\varepsilon_{4}\right) / 2\end{array}$ & $\varepsilon_{2}, \varepsilon_{4}, \varepsilon_{2}-\varepsilon_{3}, \varepsilon_{2}+\varepsilon_{4}$ & 4 & 8 \\
\hline $\begin{array}{l}\text { 20) } \varepsilon_{1}-\varepsilon_{3} \\
\left(\varepsilon_{1}+\varepsilon_{2}+\varepsilon_{3}-\varepsilon_{4}\right) / 2\end{array}$ & $\begin{array}{l}\varepsilon_{2}, \varepsilon_{2}-\varepsilon_{3}, \varepsilon_{2}-\varepsilon_{4} \\
\varepsilon_{3}-\varepsilon_{4},\left(\varepsilon_{1} \pm \varepsilon_{2}-\varepsilon_{3}-\varepsilon_{4}\right) / 2\end{array}$ & 6 & 14 \\
\hline $\begin{array}{l}\text { 21) } \varepsilon_{1}+\varepsilon_{4} \\
\left(\varepsilon_{1}+\varepsilon_{2}+\varepsilon_{3}-\varepsilon_{4}\right) / 2\end{array}$ & $\begin{array}{l}\varepsilon_{1}, \varepsilon_{2}, \varepsilon_{1}-\varepsilon_{3}, \varepsilon_{2} \pm \varepsilon_{4} \\
\left(\varepsilon_{1} \pm \varepsilon_{2}-\varepsilon_{3} \pm \varepsilon_{4}\right) / 2\end{array}$ & 9 & 20 \\
\hline $\begin{array}{l}22) \varepsilon_{2}+\varepsilon_{4}, \\
\left(\varepsilon_{1}+\varepsilon_{2}+\varepsilon_{3}-\varepsilon_{4}\right) / 2\end{array}$ & $\begin{array}{l}\varepsilon_{2}, \varepsilon_{3}, \varepsilon_{2} \pm \varepsilon_{3} \\
\varepsilon_{2}-\varepsilon_{4}, \varepsilon_{3}-\varepsilon_{4}\end{array}$ & 6 & 12 \\
\hline $\begin{array}{l}\text { 23) } \varepsilon_{1}-\varepsilon_{3} \\
\left(\varepsilon_{1}+\varepsilon_{2}+\varepsilon_{3}+\varepsilon_{4}\right) / 2\end{array}$ & $\begin{array}{l}\varepsilon_{2}, \varepsilon_{4}, \varepsilon_{2} \pm \varepsilon_{3}, \varepsilon_{2}+\varepsilon_{4}, \\
\left(\varepsilon_{1}+\varepsilon_{2}-\varepsilon_{3} \pm \varepsilon_{4}\right) / 2\end{array}$ & 7 & 16 \\
\hline $\begin{array}{l}\text { 24) } \varepsilon_{1}-\varepsilon_{4} \\
\left(\varepsilon_{1}+\varepsilon_{2}+\varepsilon_{3}+\varepsilon_{4}\right) / 2\end{array}$ & $\begin{array}{l}\varepsilon_{2}, \varepsilon_{3}, \varepsilon_{2}+\varepsilon_{3}, \varepsilon_{3}-\varepsilon_{4} \\
\varepsilon_{2} \pm \varepsilon_{4},\left(\varepsilon_{1}+\varepsilon_{2} \pm \varepsilon_{3}-\varepsilon_{4}\right) / 2\end{array}$ & 8 & 18 \\
\hline
\end{tabular}




\begin{tabular}{|c|c|c|c|}
\hline $\begin{array}{l}25) \varepsilon_{2}-\varepsilon_{4} \\
\left(\varepsilon_{1}+\varepsilon_{2}+\varepsilon_{3}+\varepsilon_{4}\right) / 2\end{array}$ & $\begin{array}{l}\varepsilon_{2}, \varepsilon_{2}+\varepsilon_{3}, \varepsilon_{2}+\varepsilon_{4}, \\
\varepsilon_{3}+\varepsilon_{4}, \varepsilon_{3}, \varepsilon_{4}, \varepsilon_{2}-\varepsilon_{3}\end{array}$ & 7 & 14 \\
\hline $\begin{array}{l}\text { 26) } \varepsilon_{1}-\varepsilon_{3}, \varepsilon_{2}-\varepsilon_{4} \\
\left(\varepsilon_{1}+\varepsilon_{2}+\varepsilon_{3}+\varepsilon_{4}\right) / 2\end{array}$ & $\begin{array}{l}\varepsilon_{2}, \varepsilon_{4}, \varepsilon_{2}+\varepsilon_{4}, \varepsilon_{2} \pm \varepsilon_{3} \\
\left(\varepsilon_{1}+\varepsilon_{2}-\varepsilon_{3} \pm \varepsilon_{4}\right) / 2\end{array}$ & 7 & 16 \\
\hline $\begin{array}{l}\text { 27) } \varepsilon_{1}-\varepsilon_{3}, \varepsilon_{2}+\varepsilon_{4} \\
\left(\varepsilon_{1}+\varepsilon_{2}+\varepsilon_{3}-\varepsilon_{4}\right) / 2\end{array}$ & $\begin{array}{l}\varepsilon_{2}, \varepsilon_{2}-\varepsilon_{3}, \varepsilon_{2}-\varepsilon_{4}, \\
\varepsilon_{3}-\varepsilon_{4},\left(\varepsilon_{1} \pm \varepsilon_{2}-\varepsilon_{3}-\varepsilon_{4}\right) / 2\end{array}$ & 6 & 14 \\
\hline $\begin{array}{l}28) \varepsilon_{1}+\varepsilon_{3}, \varepsilon_{2}+\varepsilon_{4}, \\
\left(\varepsilon_{1}-\varepsilon_{2}-\varepsilon_{3}+\varepsilon_{4}\right) / 2\end{array}$ & $\begin{array}{l}\varepsilon_{1}, \varepsilon_{4}, \varepsilon_{1}-\varepsilon_{2}, \varepsilon_{3} \pm \varepsilon_{4} \\
\left(\varepsilon_{1}-\varepsilon_{2}+\varepsilon_{3} \pm \varepsilon_{4}\right) / 2\end{array}$ & 7 & 14 \\
\hline $\begin{array}{l}29) \varepsilon_{1}-\varepsilon_{3}, \varepsilon_{2}+\varepsilon_{4}, \\
\left(\varepsilon_{1}-\varepsilon_{2}+\varepsilon_{3}-\varepsilon_{4}\right) / 2\end{array}$ & $\begin{array}{l}\varepsilon_{2}, \varepsilon_{2}-\varepsilon_{3} \\
\left(\varepsilon_{1}-\varepsilon_{2}-\varepsilon_{3} \pm \varepsilon_{4}\right) / 2\end{array}$ & 4 & 10 \\
\hline $\begin{array}{l}30) \varepsilon_{1}+\varepsilon_{4}, \varepsilon_{2}+\varepsilon_{3}, \\
\left(\varepsilon_{1}-\varepsilon_{2}+\varepsilon_{3}-\varepsilon_{4}\right) / 2\end{array}$ & $\begin{array}{l}\varepsilon_{3}, \varepsilon_{4}, \varepsilon_{2}+\varepsilon_{4}, \varepsilon_{3}+\varepsilon_{4} \\
\left(\varepsilon_{1}-\varepsilon_{2} \pm \varepsilon_{3}+\varepsilon_{4}\right) / 2\end{array}$ & 6 & 14 \\
\hline $\begin{array}{l}31) \varepsilon_{1}-\varepsilon_{3}, \varepsilon_{2}+\varepsilon_{4}, \\
\left(\varepsilon_{1}-\varepsilon_{2}+\varepsilon_{3}+\varepsilon_{4}\right) / 2\end{array}$ & $\begin{array}{l}\varepsilon_{4}, \varepsilon_{2}-\varepsilon_{3} \\
\left(\varepsilon_{1}-\varepsilon_{2}-\varepsilon_{3} \pm \varepsilon_{4}\right) / 2\end{array}$ & 4 & 10 \\
\hline $\begin{array}{l}32) \varepsilon_{1}-\varepsilon_{4}, \varepsilon_{2}+\varepsilon_{3} \\
\left(\varepsilon_{1}-\varepsilon_{2}+\varepsilon_{3}+\varepsilon_{4}\right) / 2\end{array}$ & $\begin{array}{l}\varepsilon_{3}, \varepsilon_{2}-\varepsilon_{4}, \varepsilon_{3}-\varepsilon_{4} \\
\left(\varepsilon_{1}-\varepsilon_{2} \pm \varepsilon_{3}-\varepsilon_{4}\right) / 2\end{array}$ & 5 & 12 \\
\hline $\begin{array}{l}33) \varepsilon_{1}+\varepsilon_{3}, \varepsilon_{2}+\varepsilon_{4} \\
\left(\varepsilon_{1}+\varepsilon_{2}-\varepsilon_{3}-\varepsilon_{4}\right) / 2\end{array}$ & $\begin{array}{l}\varepsilon_{1}, \varepsilon_{2}, \varepsilon_{2}-\varepsilon_{4}, \varepsilon_{3} \pm \varepsilon_{4}, \\
\varepsilon_{2}+\varepsilon_{3},\left(\varepsilon_{1}-\varepsilon_{2}+\varepsilon_{3} \pm \varepsilon_{4}\right) / 2\end{array}$ & 8 & 18 \\
\hline $\begin{array}{l}34) \varepsilon_{1}+\varepsilon_{4}, \varepsilon_{2}+\varepsilon_{3}, \\
\left(\varepsilon_{1}+\varepsilon_{2}-\varepsilon_{3}-\varepsilon_{4}\right) / 2\end{array}$ & $\begin{array}{l}\varepsilon_{1}, \varepsilon_{2}, \varepsilon_{2}-\varepsilon_{3}, \varepsilon_{3}+\varepsilon_{4}, \\
\varepsilon_{2}+\varepsilon_{4},\left(\varepsilon_{1}-\varepsilon_{2} \pm \varepsilon_{3}+\varepsilon_{4}\right) / 2\end{array}$ & 7 & 16 \\
\hline $\begin{array}{l}35) \varepsilon_{1}+\varepsilon_{3}, \varepsilon_{2}-\varepsilon_{4}, \\
\left(\varepsilon_{1}+\varepsilon_{2}-\varepsilon_{3}+\varepsilon_{4}\right) / 2\end{array}$ & $\begin{array}{l}\varepsilon_{1}, \varepsilon_{4}, \varepsilon_{1}-\varepsilon_{2}, \varepsilon_{3} \pm \varepsilon_{4} \\
\left(\varepsilon_{1}-\varepsilon_{2} \pm \varepsilon_{3} \pm \varepsilon_{4}\right) / 2\end{array}$ & 9 & 20 \\
\hline $\begin{array}{l}36) \varepsilon_{1}-\varepsilon_{4}, \varepsilon_{2}+\varepsilon_{3} \\
\left(\varepsilon_{1}+\varepsilon_{2}-\varepsilon_{3}+\varepsilon_{4}\right) / 2\end{array}$ & $\begin{array}{l}\varepsilon_{2}, \varepsilon_{2}-\varepsilon_{3}, \varepsilon_{2}-\varepsilon_{4}, \\
\varepsilon_{3}-\varepsilon_{4},\left(\varepsilon_{1} \pm \varepsilon_{2}-\varepsilon_{3}-\varepsilon_{4}\right) / 2\end{array}$ & 6 & 14 \\
\hline
\end{tabular}

\section{REFERENCES}

[1] C. A. M. André, The basic character table of the unitriangular group, J. Algebra 241 (2001), 437-471. MR 1839342 (2002e:20082)

[2] C. A. M. André and A. M. Neto, Super-characters of finite unipotent groups of types $B_{n}, C_{n}$ and $D_{n}$, J. Algebra 305 (2006), 394-429. MR2264135 (2007j:20013)

[3] M. Boyarchenko and V. Drinfeld, A motivated introduction to character sheaves and the orbit method for unipotent groups in positive characteristic, arXiv: math.RT/0609769v1.

[4] N. Bourbaki, Éléments de mathématique. Fasc. 34. Groupes et algèbres de Lie. Chapitres IV, V, VI, Actualités Sci. Indust., No. 1337, Hermann, Paris, 1968. MR.0240238 (39:1590)

[5] J. E. Humphreys, Linear algebraic groups, Grad. Texts in Math., No. 21, Springer-Verlag, New York-Heidelberg, 1975. MR0396773 (53:633)

[6] _ Introduction to Lie algebras and representation theory, 2nd ed., Grad. Texts in Math., No. 9, Springer-Verlag, New York-Berlin, 1978. MR0499562 (81b:17007)

[7] M. V. Ignat'ev and A. N. Panov, Coadjoint orbits of the group UT $(7, K)$, Fundam. Prikl. Mat. 13 (2007), no. 5, 127-159; English transl., J. Math. Sci. (N. Y.) 156 (2009), no. 2, 292-312; See also arXiv:math.RT/0603649v3. MR.2379743 (2008m:20075)

[8] _ Subregular characters of the unitriangular group over a finite field, Fundam. Prikl. Mat. 13 (2007), no. 5, 103-125; English transl., J. Math. Sci. (N. Y.) 156 (2009), no. 2, 276-291; See also arXiv: math.RT/0801.3079v2. MR2379742(2009a:20074)

[9] B Basic subsystems in the root systems $B_{n}$ and $D_{n}$ and associated coadjoint orbits, Vestn. Samar. Gos. Univ. Estestvennonauchn. Ser. 2008, no. 3, 124-148. (Russian) MR2473733 (2009i:20095)

[10] , Orthogonal subsets of classical root systems and coadjoint orbits of unipotent groups, Mat. Zametki 86 (2009), no. 1, 65-80; English transl., Math. Notes 86 (2009), no. 1-2, 65-80; See also arXiv: math.RT/0904.2841v2. MR2588639(2011a:17019)

[11] D. Kazhdan, Proof of Springer's hypothesis, Israel J. Math. 28 (1977), 272-286. MR0486181 (58:5959) 
[12] A. A. Kirillov, Lectures on the orbit method, Nauchn. Kniga, Novosibirsk, 2002; English transl., Grad. Stud. in Math., vol. 64, Amer. Math. Soc., Providence, RI, 2004. MR2069175 (2005c:22001)

[13] Unitary representations of nilpotent Lie groups, Uspekhi Mat. Nauk 17 (1962), no. 4, 57-110. (Russian) MR0142001 (25:5396)

[14] Variations on the triangular theme, Lie Groups and Lie Algebras: E. B. Dynkin's Seminar, Amer. Math. Soc. Transl. Ser. 2, vol. 169, Amer. Math. Soc., Providence, RI, 1995, pp. 43-73. MR 1364453 (97a:20072)

[15] S. Mukherjee, Coadjoint orbits for $A_{n-1}^{+}, B_{n}^{+}$and $D_{n}^{+}$, arXiv: math.RT/0501332v1. J. Lie Theory 16 (2006), 455-469. MR2248140 (2007c:17014)

[16] A. N. Panov, Involutions in $S_{n}$ and associated coadjoint orbits, Zap. Nauchn. Sem. S.-Peterburg. Otdel. Mat. Inst. Steklov. (POMI) 349 (2007), 150-173; English transl., J. Math. Sci. 151 (2008), no. 3, 3018-3031; See also math.RT/0801.3022v1. MR2742857

[17] B. Srinivasan, Representations of finite Chevalley groups, Lecture Notes in Math., vol. 764, Springer, New York-Berlin, 1979. MR0551499 (83a:20054)

[18] R. Steinberg, Lectures on Chevalley groups, Yale Univ., New Haven, Conn., 1968. MR0466335 (57:6215)

[19] _ Conjugacy classes in algebraic groups, Lecture Notes in Math., vol. 366, Springer, New York-Berlin, 1974. MR0414732 (54:2827)

Department of Algebra and Geometry, Samara State University, Ak. Pavlova 1, Samara 443011, Russia

E-mail address: mihail.ignatev@gmail.com

Received 14/APR/2010

Translated by THE AUTHOR 